\title{
An Earth Model Based on Free Oscillations and Body Waves
}

\author{
Don L. ANDERSON AND Robert S. Hart \\ Seismological Laboratory, California Institute of Technology \\ Pasadena, California 91125
}

\begin{abstract}
Several recent inversion studies have clearly indicated the lack of resolving power of the normal mode data set and the possible trade-offs among the various parameters. These studies have also shown that the final model is as dependent on the starting model as on the data set. It is therefore important to incorporate body wave data into any inversion scheme not only to gain resolution but also to reduce trade-offs between density and velocity. An earth model based on special studies of the structure of the mantle and core is inverted to be consistent with both body wave data and a representative set of normal mode observations (437 modes). The resulting model has a 40-km-thick upper mantle lithospheric lid terminating at $61 \mathrm{~km}$, with high density $\left(3.5 \mathrm{~g} / \mathrm{cm}^{3}\right)$ and seismic velocities $(8.38$ and $4.71 \mathrm{~km} / \mathrm{s})$, a pronounced upper mantle low-velocity zone (LVZ) of 180-km thickness, and transition regions of rapid velocity increase at $375-425,500-550$, and $650-675 \mathrm{~km}$. There are also anomalous gradients between 700 and $1200 \mathrm{~km}$. This model, $\mathrm{C} 2$, is slow by about 0.6 and $2-4 \mathrm{~s}$ for $P$ and $S$ waves, respectively, in comparison with body wave solutions which have a greater continental bias. The major features of the upper mantle can be explained by partial melting (LVZ) and the successive transformation of an olivinepyroxene mantle to $\beta$ spinel, $\gamma$ spinel, and garnet and further phase changes below $750 \mathrm{~km}$, In addition to the radial inhomogeneities in the upper mantle there is evidence for inhomogeneity at the base of the mantle, the top of the core, and the regions on each side of the outer core-inner core boundary.
\end{abstract}

\section{INTRODUCTION}

The normal mode data set is now adequate to determine average velocities and densities in the upper and lower mantle and the core and to resolve a certain amount of structure in these regions. However, it is not adequate to resolve details having wavelengths of the order of $100-200 \mathrm{~km}$. To resolve these features, which are particularly important in the upper mantle and the transition regions of the mantle and core, one must utilize body wave techniques, which are of a higher resolving power, including travel times, apparent velocities, amplitudes, and pulse shapes. These data by their nature and availability are more path dependent than normal modes, but it is reasonable to assume that the fine structure determined by body wave techniques is largely characteristic of the earth as a whole. The role of free oscillations then is to determine differences of the average earth from the more path specific body wave structures and to determine compatible density structures. In this spirit, we design a starting model based on highresolution body wave studies and perturb this model to fit the normal mode data set. The resulting model retains the features found by body wave studies, but the average properties in the various regions are suitably adjusted to correspond to average earth properties, as is required by the normal mode data set. This model is appropriate for discussions of gross earth chemistry and as a standard for discussing lateral variations.

Jordan and Anderson [1974] recently derived an earth model consistent with a large body of free oscillation, surface wave, and body wave data. These data tightly constrain the seismic velocities and densities in the lower mantle and outer core. However, the resolving power in the upper mantle and transition region, particularly for $P$ waves, is very poor, and the resulting model, as in all studies of this sort, is to a large extent dependent on the starting model. Although model B1, derived by Jordan and Anderson [1974], fit the available gross earth data, it has several unsatisfactory features. The upper mantle compressional velocity structure, because of the resolving power problem, appears to be inconsistent with the shear

Copyright $\odot 1976$ by the American Geophysical Union. velocity profile, which can be resolved to greater detail. In particular, the low $\boldsymbol{P}_{n}$ velocity, $7.91 \mathrm{~km} / \mathrm{s}$, is inconsistent with both the high $S_{n}$ velocity, $4.83 \mathrm{~km} / \mathrm{s}$, and the measurements of $P_{n}$ in oceanic and most continental regions. Model B1 has no $\boldsymbol{P}$ wave low-velocity layer in the upper mantle, but it does have a rather pronounced low-velocity zone (LVZ) for shear waves. Resolving power calculations indicate that an upper mantle $P$ wave LVZ cannot be resolved by the normal mode data set even though detailed body wave studies demonstrate its existence in most parts of the earth.

The low $P_{n}$ velocity and the absence of a $P$ wave LVZ are related problems, since only average properties of the upper mantle can be determined. If one accepts the $P_{n}$ data, then inversion of the same data set would yield a $P$ wave LVZ. Model Bl also gives shear wave travel times that are not consistent with recent studies [Hart, 1975; Hales and Roberts, 1970].

Recent body wave studies of the upper mantle using travel times, amplitudes, and wave shapes [Helmberger and Wiggins, 1971; Helmberger and Engen, 1974] have yielded profiles having more structure than can probably ever be resolved from gross earth data. These structures include LVZ's for both $P$ and $S$ waves and discontinuities near 375,500 , and $600 \mathrm{~km}$. Gradients between discontinuities, as well as average velocities, can also be resolved with these techniques. Although the above studies refer mostly to continental structure below North America, there is reason to believe that the major features also exist elsewhere. For example, evidence for the 375- and $600-\mathrm{km}$ discontinuities appears in great circle, mainly oceanic, dispersion data [e.g., Anderson and Toksöz, 1963] and from upper mantle reflection studies [e.g., Engdahl and Flinn, 1969; Whitcomb and Anderson, 1970]. Evidence for the 500-km discontinuity has also been discussed for oceanic regions [Whitcomb and Anderson, 1970] and for Australia [Simpson. 19731.

The interpretation of these discontinuities in terms of phase changes [Anderson, 1967a, b; Burdick and Anderson, 1975] requires that they occur everywhere, although their depths may vary slightly.

It seems appropriate therefore to adopt the high-resolution 
body wave profiles as starting models in a gross earth inversion, and to allow them to be modified as necessary to satisfy the gross earth data. We make no pretense that the fine structure in the starting and final models is required by the normal mode data set.

\section{The Starting Model}

The basic starting model is a modification of the Helmberger and Wiggins [1971] and Helmberger and Engen [1974] structures for the upper mantle, B1 for the lower mantle, and BI and Whitcomb [1973] for the core. Whitcomb [1973] constructed his core model from observed $d t / d \Delta$ 's, relative amplitudes, and arrival times of $P K P, P K i K P, S K S$, and $S K K S$, utilizing a recent mantle model [Jordan and Anderson, 1974] for the required stripping to the surface of the core. He discusses at length previous core studies. A crust and uppermost mantle model was derived which is an average of the tectonic subdivisions of the earth. It includes a $3-\mathrm{km}$-thick water layer in order to overcome the objections of Hales [1974]. It has a 40-km-thick lid (the mantle part of the lithosphere), a 58-kmthick lithosphere, pronounced low-velocity zones for both $\boldsymbol{P}$ and $S$, and discontinuities or rapid increases in velocity, near 375,500 , and $670 \mathrm{~km}$. The latter discontinuity was made sharp in order to satisfy $P^{\prime} P^{\prime}$ precursor reflection data [Engdahl and Flinn. 1969; Whitcomb and Anderson, 1970].

Model B1 of Jordan and Anderson [1974] represented the 'shortest smooth perturbation' from a simple initial model that incorporated the major seismic discontinuities (400 and 600 $\mathrm{km}$ ) found from previous body wave and surface wave studies [Anderson and Toksöz, 1963; Niazi and Anderson, 1965; Julian and Anderson, 1968; Johnson, 1967] and that upon inversion satisfied the normal mode data set of Dziewonski and Gilbert [1972] and a large body of supplementary data including travel times, apparent velocities, and group velocities. The starting model had an adiabatic and homogeneous lower mantle and outer core. The starting, or initial, model for the present study incorporates fine structure of the upper mantle [Helmberger and Wiggins, 1971; Helmberger and Engen, 1974], uppermost lower mantle [Hart, 1975], and core [Whitcomb, 1973] which is unresolvable by the normal mode data set. In addition, we modified the starting $V_{p}$ model to be consistent with the $P_{n}$ data. The starting density model contains discontinuities in the upper mantle at the depths of the seismic discontinuities.

It should be emphasized that in linear inversion the starting model is as important as the data set. Our starting model incorporates features found by techniques which have an intrinsic greater resolving power than the gross earth data set itself. The inversion technique that we used is identical to that described by Jordan and Anderson [1974]. For the forward part of the calculations we used programs written by Martin Smith. The radius of the core was fixed at $3485 \mathrm{~km}$, the value determined by Jordan and Anderson [1974] and verified by Engdahl and Johnson [1974]. This core radius is also consistent with the solutions of Hales and Roberts [1970] and Gilbert and Dziewonski [1975]. It is about $12 \mathrm{~km}$ larger than earlier determinations, such as that of Jeffreys and Bullen [1940], but agrees with one of the solutions of Hales and Roberts [1970].

As a first step we inverted the toroidal mode data for shear velocity and density, thus removing the coupling between $V_{p}$ and $V_{\mathrm{s}}$. We then inverted using a combination of toroidal modes and the spheroidal modes that are particularly sensitive to shear velocity, checking against $S c S-S$ and the shape of the shear wave travel time curve at various stages. Once these data are satisfied, we have an accurate shear velocity profile and a first approximation to the density perturbation. Modes that are sensitive to compressional velocity and density were then inverted for these parameters, with checks being made at various stages of the iterative process against body wave data such as $P c P-P, P$ wave residuals, and differential core times. The perturbations in density at this stage affected the fits of the toroidal modes, since they are slightly dependent on density. They were consequently reinverted. Modes that are strongly affected by all three parameters were inverted at the end of each iteration cycle in order to decrease the coupling between parameters. More and more higher spheroidal overtones were incorporated into the data set as the number of iterations increased, until it became clear that the fit to the more accurate and complete lower-order data was starting to degrade, while the model itself was almost indistinguishable from earlier iterations. Satisfactory convergence was achieved after about eight iteration cycles and a total of 32 iterations on various subsets of the data. All the modes and body wave parameters were then recomputed. This procedure, although cumbersome, seems preferable to inverting simultaneously for all parameters by using all the normal mode data with equal weight.

\section{The Normal Mode Data SeT}

For the first several iterations we used the same 177 modes as used in the study of Jordan and Anderson [1974]. This includes the first five radial modes, the fundamental spheroidal modes ${ }_{0} S_{2}-_{0} S_{63}$, the fundamental toroidal modes ${ }_{0} T_{2}-_{0} T_{48}, 56$ spheroidal overtones, and nine toroidal overtones. Most of these data are from Dziewonski and Gilbert [1973] and Gilbert and Dziewonski [1975]. Gilbert and Dziewonski [1975] have recently presented the results of a new analysis and have tabulated what they feel to be the 'best' observation for each mode. However, their criterion for best is model dependent.

In the final inversions we used 400 representative modes including 148 toroidal overtones up to ${ }_{7} T_{49}$ and 136 spheroidal overtones up to ${ }_{5} S_{35}$. Eight radial modes were used. The data are from Dziewonski and Gilbert [1973], Gilbert and Dziewonski [1975], Bolt and Currie [1975], Mendiguren [1973], Derr [1969], and H. Kanamori (unpublished results, 1975). Unfortunately, the techniques used by Mendiguren [1973] and Gilbert and Dziewonski [1975] do not yield reliable estimates of the errors. We follow the latter authors in assuming that $0.05 \%$ is a minimum error but otherwise adopt the published error estimates. In many cases the tabulated error is much less than one would infer by comparing the various data sets. The eigenperiods and estimates of their errors are tabulated in Table 2.

For the toroidal data set we have used essentially the same modes as those used by Gilbert and Dziewonski [1975] except that we have deleted the data of Brune and Gilbert [1974] which have large uncertainties $(\sim 0.40 \%)$, are not fit well by the Gilbert-Dziewonski models, and represent properties only over a very short arc length of the earth's surface. The remaining data include the fundamental and first seven toroidal overtones having periods greater than $73 \mathrm{~s}$. This process eliminates 156 modes from the Gilbert-Dziewonski toroidal data set.

Although they are not used in the inversion, we have spotchecked modes in each overtone group up to the 22 nd spheroidal overtone. Agreement is satisfactory.

\section{RESULTS OF THE INVERSION}

The final model, designated $\mathrm{C} 2$, fits the toroidal data set, 192 modes, with an average error of $0.09 \%$ and the radial-spheroidal data set, 208 modes, with an average error of $0.07 \%$. A 
TABLE 1. Summary of Fit of C2 to Normal Mode Data

\begin{tabular}{|c|c|}
\hline Modes & Error, \% \\
\hline 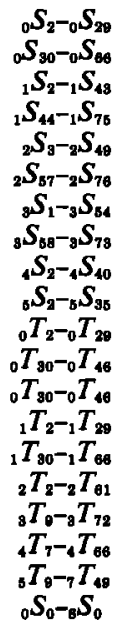 & $\begin{array}{l}0.03 \\
0.08 \\
0.10 \\
0.07 \\
0.07 \\
0.15 \\
0.05 \\
0.11 \\
0.08 \\
0.09 \\
0.04 \\
0.17 \\
0.08 \\
0.11 \\
0.07 \\
0.08 \\
0.08 \\
0.16 \\
0.09 \\
0.05\end{array}$ \\
\hline
\end{tabular}

Average error for radial-spheroidal data set is 0.07 ; average error for toroidal data set is 0.09 .

*Includes traveling wave data sets.

summary of the fit is given in Table 1 . The complete data set along with computed periods for $\mathrm{C} 2$ is given in Table 2. The fits to ${ }_{0} S_{2-o} S_{29}$ and ${ }_{0} T_{2-0} T_{28}$, the fundamental modes, are $0.03 \%$ and $0.05 \%$, respectively. These are generally the best-excited and most accurately determined modes, and it is important that they be fit well. More determinations have also been made of these modes, and they therefore represent a better gross earth average than some of the higher modes for which, in many cases, only a single observation is available. Fifty-two of the modes, or $13 \%$, are fit to better than 1 part in 10,000 , and 282 modes, or $71 \%$, are fit to 1 part in $1000 ; 244$ modes, or $61 \%$, are fit to 1 standard deviation, and 343 , or $86 \%$, are fit to 2 standard deviations. Although this represents a good overall fit, it is not as good as it should be if all the data are independent and if the error estimates are reliable. In spite of the great increase in the normal mode data set there are still some modes whose identification or period assignment is questionable. Of the present 400 -mode data set there are 40 modes that are not fit well ( $>0.15 \%$ error) by either $\mathrm{C} 2$ or $1066 \mathrm{~B}$ of

TABLE 2. Observed and Computed Eigenperiods

\begin{tabular}{ccccc}
\hline Mode & Data, s & Error, $\%$ & \multicolumn{1}{c}{ C2 } & Difference, $\%$ \\
\hline${ }_{0} T_{2}$ & 2636.38 & 0.08 & 2630.18 & 0.24 \\
${ }_{0} T_{3}$ & 1702.51 & 0.15 & 1702.30 & 0.01 \\
${ }_{0} T_{4}$ & 1303.60 & 0.07 & 1303.63 & 0.00 \\
${ }_{0} T_{6}$ & 1075.20 & 0.09 & 1075.53 & -0.03 \\
${ }_{0} T_{6}$ & 925.36 & 0.09 & 925.55 & -0.02 \\
${ }_{0} T_{7}$ & 817.92 & 0.08 & 818.04 & -0.01 \\
${ }_{0} T_{8}$ & 736.86 & 0.05 & 736.39 & 0.06 \\
${ }_{0} T_{9}$ & 671.80 & 0.06 & 671.76 & 0.01 \\
& & & & \\
${ }_{0} T_{10}$ & 619.02 & 0.05 & 619.03 & -0.00 \\
${ }_{0} T_{11}$ & 574.62 & 0.08 & 574.99 & -0.06 \\
${ }_{0} T_{12}$ & 536.93 & 0.05 & 537.52 & -0.11 \\
${ }_{0} T_{1 \mathrm{~s}}$ & 504.94 & 0.08 & 505.16 & -0.04 \\
${ }_{0} T_{14}$ & 476.64 & 0.08 & 476.86 & -0.04 \\
${ }_{0} T_{18}$ & 451.83 & 0.06 & 451.83 & -0.00 \\
${ }_{0} T_{10}$ & 429.50 & 0.07 & 429.52 & 0.00 \\
${ }_{0} T_{17}$ & 409.61 & 0.05 & 409.46 & 0.04 \\
${ }_{0} T_{18}$ & 391.16 & 0.10 & 391.32 & -0.04 \\
${ }_{0} T_{19}$ & 374.76 & 0.05 & 374.80 & -0.01 \\
& & & &
\end{tabular}

TABLE 2. (continued)

\begin{tabular}{|c|c|c|c|c|}
\hline Mode & Data, s & Error, \% & C2 & Difference, $\%$ \\
\hline $\begin{array}{l}{ }_{0}^{0} T_{20} \\
{ }_{0} T_{21} \\
{ }_{0} T_{22} \\
{ }_{0}^{0} T_{2 \mathrm{~s}} \\
{ }_{0} T_{24} \\
{ }_{0}^{0} T_{2 \mathrm{~s}} \\
{ }_{0}^{0} T_{2 \mathrm{~g}} \\
{ }_{0}^{0} T_{27} \\
{ }_{0} T_{2 \mathrm{~g}} \\
{ }_{0}^{0} T_{29}\end{array}$ & $\begin{array}{l}359.59 \\
345.70 \\
333.15 \\
321.21 \\
310.18 \\
299.51 \\
290.26 \\
281.21 \\
272.60 \\
264.66\end{array}$ & $\begin{array}{l}0.08 \\
0.05 \\
0.13 \\
0.09 \\
0.08 \\
0.10 \\
0.06 \\
0.16 \\
0.27 \\
0.05\end{array}$ & $\begin{array}{l}359.68 \\
345.79 \\
332.97 \\
321.10 \\
310.06 \\
299.78 \\
290.17 \\
281.16 \\
272.70 \\
264.75\end{array}$ & $\begin{array}{r}-0.03 \\
-0.03 \\
0.05 \\
0.04 \\
0.04 \\
-0.09 \\
0.03 \\
0.02 \\
-0.04 \\
-0.03\end{array}$ \\
\hline $\begin{array}{l}{ }_{0} T_{30} \\
{ }_{0} T_{31} \\
{ }_{0} T_{32} \\
{ }_{0} T_{39} \\
{ }_{0} T_{38} \\
{ }_{0}^{0} T_{36} \\
{ }_{0}^{0} T_{37} \\
{ }_{0} T_{38} \\
{ }_{0} T_{38}\end{array}$ & $\begin{array}{l}257.29 \\
250.14 \\
243.43 \\
237.37 \\
231.29 \\
219.69 \\
213.89 \\
209.83 \\
204.27\end{array}$ & $\begin{array}{l}0.15 \\
0.04 \\
0.07 \\
0.10 \\
0.10 \\
0.11 \\
0.10 \\
0.28 \\
0.05\end{array}$ & $\begin{array}{l}257.25 \\
250.16 \\
243.46 \\
237.10 \\
231.07 \\
219.89 \\
214.69 \\
209.73 \\
205.00\end{array}$ & $\begin{array}{r}0.02 \\
-0.01 \\
0.01 \\
0.11 \\
0.09 \\
-0.09 \\
-0.37 \\
0.05 \\
-0.36\end{array}$ \\
\hline $\begin{array}{l}{ }_{0} T_{40} \\
{ }_{0} T_{41} \\
{ }_{0} T_{42} \\
{ }_{0} T_{48} \\
{ }_{0} T_{44} \\
{ }_{0} T_{45} \\
{ }_{0} T_{40}\end{array}$ & $\begin{array}{l}199.96 \\
195.88 \\
191.26 \\
187.40 \\
183.78 \\
180.25 \\
176.85\end{array}$ & $\begin{array}{l}0.19 \\
0.22 \\
0.13 \\
0.26 \\
0.15 \\
0.05 \\
0.05\end{array}$ & $\begin{array}{l}200.48 \\
196.15 \\
192.00 \\
188.02 \\
184.21 \\
180.54 \\
177.02\end{array}$ & $\begin{array}{l}-0.26 \\
-0.14 \\
-0.38 \\
-0.32 \\
-0.23 \\
-0.16 \\
-0.09\end{array}$ \\
\hline $\begin{array}{l}{ }_{1} T_{2} \\
{ }_{1} T_{3} \\
{ }_{1} T_{4} \\
{ }_{1} T_{6} \\
{ }_{1} T_{7} \\
{ }_{1} T_{8} \\
{ }_{1} T_{9}\end{array}$ & $\begin{array}{l}756.57 \\
695.18 \\
629.98 \\
519.09 \\
475.17 \\
438.49 \\
407.73\end{array}$ & $\begin{array}{l}0.08 \\
0.07 \\
0.10 \\
0.06 \\
0.13 \\
0.05 \\
0.10\end{array}$ & $\begin{array}{l}756.22 \\
693.65 \\
629.61 \\
518.53 \\
474.74 \\
438.17 \\
407.57\end{array}$ & $\begin{array}{l}0.05 \\
0.22 \\
0.06 \\
0.11 \\
0.09 \\
0.07 \\
0.04\end{array}$ \\
\hline $\begin{array}{l}{ }_{1} T_{10} \\
{ }_{1} T_{11} \\
{ }_{1} T_{12} \\
{ }_{1} T_{18} \\
{ }_{1} T_{18}\end{array}$ & $\begin{array}{l}381.65 \\
359.14 \\
339.54 \\
322.84 \\
280.59\end{array}$ & $\begin{array}{l}0.10 \\
0.05 \\
0.06 \\
0.12 \\
0.06\end{array}$ & $\begin{array}{l}381.68 \\
359.45 \\
340.05 \\
322.91 \\
281.35\end{array}$ & $\begin{array}{l}-0.01 \\
-0.09 \\
-0.15 \\
-0.02 \\
-0.27\end{array}$ \\
\hline $\begin{array}{l}{ }_{1} T_{20} \\
{ }_{1} T_{24} \\
{ }_{1} T_{25} \\
{ }_{1} T_{28} \\
{ }_{1} T_{20}\end{array}$ & $\begin{array}{l}240.98 \\
211.95 \\
205.85 \\
200.27 \\
185.34\end{array}$ & $\begin{array}{l}0.09 \\
0.05 \\
0.05 \\
0.05 \\
0.05\end{array}$ & $\begin{array}{l}241.29 \\
212.22 \\
206.16 \\
200.51 \\
185.57\end{array}$ & $\begin{array}{l}-0.13 \\
-0.13 \\
-0.15 \\
-0.12 \\
-0.12\end{array}$ \\
\hline $\begin{array}{l}{ }_{1} T_{90} \\
{ }_{1} T_{31} \\
{ }_{1} T_{38} \\
{ }_{1} T_{34} \\
{ }_{1} T_{96} \\
{ }_{1} T_{30} \\
{ }_{1} T_{97} \\
{ }_{1} T_{38} \\
{ }_{1} T_{38}\end{array}$ & $\begin{array}{l}180.80 \\
176.85 \\
169.27 \\
165.72 \\
162.36 \\
159.11 \\
156.08 \\
153.17 \\
150.28\end{array}$ & $\begin{array}{l}0.06 \\
0.07 \\
0.05 \\
0.05 \\
0.05 \\
0.05 \\
0.05 \\
0.08 \\
0.07\end{array}$ & $\begin{array}{l}181.16 \\
177.00 \\
169.32 \\
165.78 \\
162.41 \\
159.20 \\
156.14 \\
153.21 \\
150.41\end{array}$ & $\begin{array}{l}-0.20 \\
-0.08 \\
-0.03 \\
-0.04 \\
-0.03 \\
-0.06 \\
-0.04 \\
-0.02 \\
-0.08\end{array}$ \\
\hline $\begin{array}{l}{ }_{1} T_{40} \\
{ }_{1} T_{41} \\
{ }_{1} T_{42} \\
{ }_{1} T_{43} \\
{ }_{1} T_{44} \\
{ }_{1} T_{45} \\
{ }_{1} T_{48} \\
{ }_{1} T_{47} \\
{ }_{1} T_{48}\end{array}$ & $\begin{array}{l}147.68 \\
145.12 \\
142.66 \\
140.23 \\
137.96 \\
135.64 \\
133.63 \\
131.59 \\
129.56\end{array}$ & $\begin{array}{l}0.05 \\
0.07 \\
0.06 \\
0.08 \\
0.06 \\
0.24 \\
0.07 \\
0.17 \\
0.06\end{array}$ & $\begin{array}{l}147.72 \\
145.14 \\
142.67 \\
140.29 \\
138.00 \\
135.79 \\
133.66 \\
131.60 \\
129.62\end{array}$ & $\begin{array}{l}-0.03 \\
-0.02 \\
-0.01 \\
-0.04 \\
-0.03 \\
-0.11 \\
-0.02 \\
-0.01 \\
-0.04\end{array}$ \\
\hline $\begin{array}{l}{ }_{1} T_{80} \\
{ }_{1} T_{61} \\
{ }_{1} T_{82} \\
{ }_{1} T_{54} \\
{ }_{1} T_{67}\end{array}$ & $\begin{array}{l}125.92 \\
124.13 \\
122.26 \\
118.96 \\
114.41\end{array}$ & $\begin{array}{l}0.08 \\
0.43 \\
0.14 \\
0.13 \\
0.12\end{array}$ & $\begin{array}{l}125.83 \\
124.03 \\
122.28 \\
118.94 \\
114.27\end{array}$ & $\begin{array}{r}0.07 \\
0.08 \\
-0.02 \\
0.02 \\
0.12\end{array}$ \\
\hline
\end{tabular}


TABLE 2. (continued)

\begin{tabular}{|c|c|c|c|c|c|c|c|c|c|}
\hline Mode & Data,s & Error, \% & $\mathrm{C} 2$ & Difference, \% & Mode & Data, s & Error, \% & $\mathrm{C} 2$ & Difference, $\%$ \\
\hline${ }_{1} T_{\mathrm{sB}}$ & 112.92 & 0.12 & 112.81 & 0.10 & ${ }_{4} T_{18}$ & 174.72 & 0.19 & 175.34 & -0.35 \\
\hline \multirow[t]{2}{*}{${ }_{1} T_{s 0}$} & 111.40 & 0.09 & 111.38 & 0.02 & $T_{20}$ & 155.64 & 0.19 & 155.80 & -0.10 \\
\hline & & & & & ${ }_{4} T_{22}$ & 147.47 & 0.19 & 147.17 & 0.20 \\
\hline${ }_{1} T_{80}$ & 110.24 & 0.13 & 109.98 & 0.23 & ${ }_{4} T_{23}$ & 143.67 & 0.19 & 143.24 & 0.30 \\
\hline${ }_{1} T_{62}$ & 107.44 & 0.13 & 107.31 & 0.13 & $T_{25}$ & 136.30 & 0.20 & 136.11 & 0.14 \\
\hline${ }_{1} T_{64}$ & 104.94 & 0.13 & 104.76 & 0.17 & $T_{n 7}$ & 130.03 & 0.23 & 129.80 & 0.17 \\
\hline \multirow[t]{2}{*}{${ }_{1} T_{66}$} & 102.59 & 0.14 & 102.34 & 0.25 & & & & & \\
\hline & & & & & ${ }_{4} T_{40}$ & 101.27 & 0.30 & 101.32 & -0.05 \\
\hline${ }_{2} T_{2}$ & 447.30 & 0.09 & 448.21 & -0.20 & ${ }_{4} T_{46}$ & 93.79 & 0.10 & 93.88 & -0.09 \\
\hline${ }_{2} T$ & 419.38 & 0.09 & 420.34 & -0.23 & ${ }_{4} T_{48}$ & 89.82 & 0.10 & 89.98 & -0.17 \\
\hline${ }_{2} T_{5}$ & 401.82 & 0.09 & 402.63 & -0.20 & $T_{60}$ & 87.46 & 0.09 & 87.56 & -0.12 \\
\hline$T_{7}$ & 363.65 & 0.07 & 363.43 & 0.06 & $T_{54}$ & 82.95 & 0.10 & 83.13 & -0.22 \\
\hline${ }_{2} T_{8}$ & 343.34 & 0.06 & 343.43 & -0.03 & $T_{\text {eg }}$ & 74.72 & 0.09 & 74.68 & 0.04 \\
\hline$T_{17}$ & 219.95 & 0.06 & 219.97 & -0.01 & $T_{64}$ & 73.79 & 0.09 & 73.86 & -0.10 \\
\hline${ }_{2} T_{18}$ & 211.90 & 0.06 & 212.07 & -0.08 & $T_{\mathrm{es}}$ & 72.94 & 0.10 & 73.05 & -0.15 \\
\hline${ }_{2} T_{18}$ & 204.63 & 0.10 & 204.83 & -0.10 & ${ }_{4} T_{\theta e}$ & 72.28 & 0.10 & 72.26 & 0.03 \\
\hline${ }_{2} T_{21}$ & 191.91 & 0.06 & 191.97 & -0.03 & ${ }_{8} T_{0}$ & 174.33 & 0.10 & 174.67 & -0.19 \\
\hline${ }_{2} T_{22}$ & 186.19 & 0.06 & 186.22 & -0.02 & ${ }_{3} T_{10}$ & 171.89 & 0.08 & 172.17 & -0.16 \\
\hline${ }_{2} T_{25}$ & 171.12 & 0.12 & 171.14 & -0.01 & ${ }_{8} T_{16}$ & 157.57 & 0.10 & 157.65 & -0.05 \\
\hline${ }_{2} T_{26}$ & 166.50 & 0.07 & 166.72 & -0.13 & ${ }_{6} T_{9 a}$ & 97.11 & 0.09 & 97.11 & 0.00 \\
\hline${ }_{2} T_{27}$ & 162.58 & 0.09 & 162.54 & 0.02 & ${ }_{6} T_{40}$ & 94.12 & 0.08 & 94.12 & 0.00 \\
\hline${ }_{3} T_{28}$ & 158.43 & 0.05 & 158.59 & -0.10 & ${ }_{6} T_{44}$ & 88.64 & 0.09 & 88.69 & -0.05 \\
\hline \multirow{2}{*}{$T_{2 g}$} & 154.70 & 0.06 & 154.85 & -0.10 & ${ }_{8} T_{46}$ & 87.47 & 0.09 & 87.43 & 0.05 \\
\hline & & & & & ${ }_{5} T_{50}$ & 81.60 & 0.10 & 81.65 & -0.06 \\
\hline${ }_{2} T_{31}$ & 147.71 & 0.06 & 147.93 & -0.15 & ${ }_{5} T_{56}$ & 76.52 & 0.09 & 76.61 & -0.12 \\
\hline${ }_{3} T_{32}$ & 144.59 & 0.06 & 144.72 & -0.09 & ${ }_{5} T_{57}$ & 74.75 & 0.09 & 74.78 & -0.04 \\
\hline${ }_{2} T_{24}$ & 138.62 & 0.06 & 138.74 & -0.08 & & & & & \\
\hline${ }_{2} T_{36}$ & 135.73 & 0.06 & 135.94 & -0.16 & ${ }_{8} T_{s 4}$ & 97.13 & 0.10 & 97.06 & 0.07 \\
\hline${ }_{2} T_{30}$ & 133.14 & 0.06 & 133.28 & -0.10 & ${ }_{9} T_{\mathrm{sb}}$ & 95.46 & 0.09 & 95.42 & 0.04 \\
\hline${ }_{2} T_{37}$ & 130.51 & 0.06 & 130.72 & -0.16 & ${ }_{0} T_{41}$ & 86.70 & 0.09 & 86.77 & -0.09 \\
\hline${ }_{2} T_{38}$ & 128.17 & 0.08 & 128.28 & -0.09 & ${ }_{8} T_{42}$ & 85.35 & 0.09 & 85.50 & -0.17 \\
\hline \multirow{2}{*}{${ }_{2} T_{8 \mathrm{~B}}$} & 125.71 & 0.06 & 125.93 & -0.18 & ${ }_{6} T_{46}$ & 81.85 & 0.10 & 81.90 & -0.05 \\
\hline & & & & & ${ }_{6} T_{49}$ & 77.65 & 0.09 & 77.59 & 0.08 \\
\hline${ }_{2} T_{40}$ & 123.56 & 0.06 & 123.68 & -0.10 & ${ }_{6} T_{s 3}$ & 73.89 & 0.09 & 73.78 & 0.15 \\
\hline${ }_{2} T_{41}$ & 121.57 & 0.05 & 121.53 & 0.03 & & & & & \\
\hline${ }_{2} T_{42}$ & 119.33 & 0.14 & 119.46 & -0.11 & ${ }_{7} T_{8}$ & 129.67 & 0.39 & 129.27 & 0.31 \\
\hline${ }_{2} T_{44}$ & 115.49 & 0.06 & 115.55 & -0.06 & ${ }_{7} T_{17}$ & 118.57 & 0.13 & 118.60 & -0.03 \\
\hline${ }_{2} T_{45}$ & 113.57 & 0.06 & 113.72 & -0.13 & ${ }_{7} T_{18}$ & 115.58 & 0.14 & 115.69 & -0.10 \\
\hline${ }_{2} T_{47}$ & 110.22 & 0.06 & 110.25 & -0.02 & ${ }_{7} T_{28}$ & 101.15 & 0.13 & 101.42 & -0.26 \\
\hline \multirow[t]{2}{*}{${ }_{2} T_{49}$} & 106.98 & 0.06 & 107.03 & -0.04 & ${ }_{7} T_{29}$ & 99.53 & 0.13 & 99.74 & -0.21 \\
\hline & & & & & ${ }_{7} T_{30}$ & 97.93 & 0.13 & 98.05 & -0.12 \\
\hline${ }_{2} T_{61}$ & 104.01 & 0.06 & 104.03 & -0.02 & ${ }_{7} T_{34}$ & 91.46 & 0.14 & 91.40 & 0.06 \\
\hline${ }_{2} T_{\mathrm{sa}}$ & 102.60 & 0.06 & 102.62 & -0.02 & $T_{38}$ & 85.45 & 0.13 & 85.49 & -0.05 \\
\hline${ }_{2} T_{34}$ & 99.93 & 0.06 & 99.92 & 0.01 & ${ }_{7} T_{40}$ & 82.84 & 0.14 & 82.89 & -0.06 \\
\hline${ }_{2} T_{55}$ & 98.61 & 0.06 & 98.65 & -0.04 & ${ }_{7} T_{40}$ & 76.18 & 0.13 & 76.19 & -0.02 \\
\hline${ }_{2} T_{30}$ & 95.08 & 0.06 & 95.04 & 0.04 & ${ }_{79}$ & 73.36 & 0.15 & 73.32 & 0.05 \\
\hline \multirow[t]{2}{*}{${ }_{2} T_{01}$} & 91.85 & 0.07 & 91.76 & 0.10 & & & & & \\
\hline & & & & & ${ }_{0} S_{0}$ & 1227.64 & 0.06 & 1228.47 & -0.07 \\
\hline${ }_{\mathrm{a}} T_{\mathrm{g}}$ & 259.26 & 0.12 & 259.38 & -0.05 & ${ }_{1} S_{0}$ & 613.59 & 0.05 & 613.91 & -0.05 \\
\hline$T_{11}$ & 240.50 & 0.10 & 240.80 & -0.13 & ${ }_{2} S_{0}$ & 398.55 & 0.05 & 398.58 & -0.01 \\
\hline${ }_{8} T_{17}$ & 189.97 & 0.13 & 190.77 & -0.42 & ${ }_{8} S_{0}$ & 305.84 & 0.05 & 306.01 & -0.05 \\
\hline${ }_{8} T_{16}$ & 184.09 & 0.09 & 184.28 & -0.10 & $S_{0}$ & 243.59 & 0.05 & 243.44 & 0.06 \\
\hline${ }_{3} T_{19}$ & 178.17 & 0.09 & $178.3 j$ & -0.09 & ${ }_{B} S_{0}$ & 204.61 & 0.05 & 204.70 & -0.05 \\
\hline${ }_{9} T_{20}$ & 172.74 & 0.06 & 172.87 & -0.07 & $S_{0}$ & 174.25 & 0.09 & 174.10 & 0.09 \\
\hline$T_{\mathrm{a1}}$ & 167.69 & 0.06 & 167.84 & -0.09 & $S_{0}$ & 134.65 & 0.05 & 134.66 & 0.00 \\
\hline${ }_{9} T_{2 \mathrm{~g}}$ & 158.54 & 0.06 & 158.81 & -0.17 & & & & & \\
\hline${ }_{3} T_{24}$ & 154.81 & 0.12 & 154.72 & 0.06 & ${ }_{0} S_{2}$ & 3233.26 & 0.06 & 3231.89 & 0.04 \\
\hline${ }_{\mathrm{g}} T_{2 \mathrm{~s}}$ & 150.66 & 0.05 & 150.87 & -0.14 & ${ }_{0} S_{3}$ & 2133.58 & 0.11 & 2133.63 & 0.00 \\
\hline \multirow[t]{2}{*}{${ }_{3}^{802} T_{28}$} & 137.24 & 0.07 & 137.35 & -0.08 & ${ }_{0} S_{4}$ & 1545.60 & 0.05 & 1545.43 & 0.01 \\
\hline & & & & & $S_{s}$ & 1190.12 & 0.05 & 1190.11 & 0.00 \\
\hline${ }_{8} T_{39}$ & 126.16 & 0.06 & 126.21 & -0.04 & $S_{8}$ & 963.17 & 0.05 & 963.46 & -0.03 \\
\hline${ }_{3} T_{34}$ & 123.75 & 0.06 & 123.72 & 0.02 & $S_{7}$ & 811.45 & 0.05 & 812.06 & -0.08 \\
\hline${ }_{8} T_{37}$ & 116.89 & 0.06 & 116.87 & 0.02 & ${ }_{0} S_{\mathrm{B}}$ & 707.64 & 0.05 & 707.68 & 0.00 \\
\hline${ }_{8} T_{41}$ & 108.87 & 0.06 & 108.94 & -0.06 & ${ }_{0} S_{0}$ & 633.95 & 0.05 & 633.73 & 0.03 \\
\hline$T_{47}$ & 99.08 & 0.06 & 99.08 & 0.00 & & & & & \\
\hline${ }_{3} T_{51}$ & 93.67 & 0.09 & 93.56 & 0.12 & ${ }_{0} S_{10}$ & 580.06 & 0.05 & 579.32 & 0.13 \\
\hline$T_{59}$ & 84.35 & 0.09 & 84.35 & 0.00 & ${ }_{0} S_{11}$ & 536.98 & 0.05 & 537.04 & -0.01 \\
\hline & 78.69 & 0.10 & 78.70 & -0.01 & ${ }_{0} S_{12}$ & 502.33 & 0.06 & 502.45 & -0.02 \\
\hline$T_{72}$ & 73.16 & 0.10 & 73.16 & 0.00 & $S_{13}$ & 473.17 & 0.06 & 473.27 & -0.02 \\
\hline & & & & & ${ }_{0} S_{14}$ & 448.20 & 0.05 & 448.11 & 0.02 \\
\hline${ }_{4} T_{7}$ & 216.81 & 0.18 & 217.27 & -0.21 & ${ }_{0} S_{16}$ & 426.06 & 0.05 & 426.11 & -0.01 \\
\hline${ }_{4} T_{21}$ & 199.74 & 0.19 & 200.99 & -0.17 & ${ }_{0} S_{16}$ & 406.75 & 0.05 & 406.69 & 0.01 \\
\hline$T$ & 184.86 & 0.19 & 185.44 & -0.31 & ${ }_{0} S_{17}$ & 389.32 & 0.05 & 389.42 & -0.03 \\
\hline
\end{tabular}


TABLE 2. (continued)

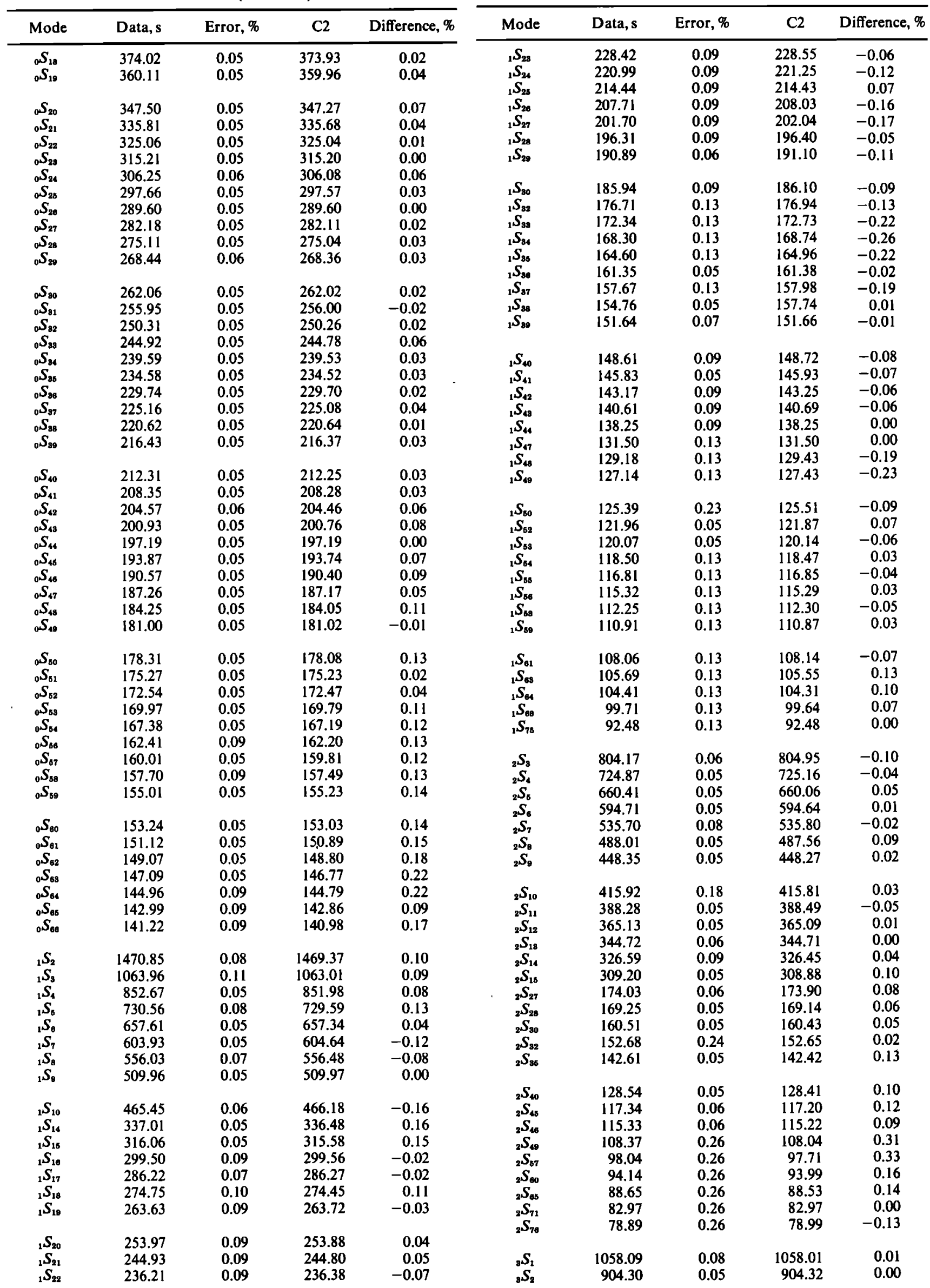


TABLE 2. (continued)

\begin{tabular}{|c|c|c|c|c|c|c|c|c|c|}
\hline Mode & Data, $s$ & Error, \% & $\mathrm{C} 2$ & Difference, $\%$ & Mode & Data, s & Error, \% & $\mathrm{C} 2$ & Difference, $\%$ \\
\hline$S_{\mathrm{a}}$ & 392.33 & 0.05 & 392.00 & 0.08 & r & & & & \\
\hline${ }_{3} S_{7}$ & 372.05 & 0.05 & 372.03 & 0.01 & ${ }_{10} S_{2}$ & 247.74 & 0.05 & 246.80 & 0.38 \\
\hline${ }_{8} S_{\mathrm{g}}$ & 354.56 & 0.05 & 354.39 & 0.05 & ${ }_{10} S_{18}$ & 134.95 & 0.05 & 134.88 & 0.05 \\
\hline \multirow[t]{2}{*}{$S_{0}$} & 338.90 & 0.08 & 338.53 & 0.11 & ${ }_{11} S_{1}$ & 271.36 & 0.09 & 271.47 & -0.04 \\
\hline & & & & & ${ }_{11} S_{24}$ & 104.43 & 0.05 & 104.63 & -0.19 \\
\hline${ }_{2} S_{10}$ & 323.94 & 0.06 & 323.92 & 0.01 & ${ }_{12} S_{7}$ & 170.69 & 0.05 & 171.02 & -0.19 \\
\hline${ }_{3} S_{11}$ & 310.27 & 0.08 & 310.19 & 0.02 & ${ }_{18} S_{1}$ & 222.69 & 0.09 & 222.82 & -0.06 \\
\hline${ }_{8} S_{12}$ & 297.41 & 0.08 & 297.22 & 0.06 & ${ }_{13} S_{10}$ & 103.43 & 0.06 & 103.52 & -0.09 \\
\hline${ }_{8} S_{18}$ & 285.08 & 0.08 & 284.93 & 0.05 & ${ }_{14} S_{4}$ & 180.81 & 0.13 & 180.43 & 0.20 \\
\hline$S_{14}$ & 273.35 & 0.05 & 273.29 & 0.02 & ${ }_{15} S_{8}$ & 165.83 & 0.05 & 165.63 & 0.12 \\
\hline${ }_{8} S_{18}$ & 251.98 & 0.05 & 251.98 & 0.00 & ${ }_{15} S_{10}$ & 100.77 & 0.05 & 100.90 & -0.13 \\
\hline${ }_{8} S_{17}$ & 242.43 & 0.05 & 242.29 & 0.06 & ${ }_{18} S_{2}$ & 175.29 & 0.05 & 175.81 & -0.30 \\
\hline${ }_{8} S_{18}$ & 233.29 & 0.05 & 233.23 & 0.03 & ${ }_{10} S_{10}$ & 118.62 & 0.09 & 118.58 & 0.03 \\
\hline \multirow[t]{2}{*}{$S_{10}$} & 224.91 & 0.05 & 224.76 & 0.07 & ${ }_{17} S_{15}$ & 100.48 & 0.09 & 100.43 & 0.05 \\
\hline & & & & & ${ }_{18} S_{\mathrm{g}}$ & 145.28 & 0.05 & 145.27 & 0.00 \\
\hline${ }_{8} S_{20}$ & 216.95 & 0.09 & 216.84 & 0.05 & ${ }_{18} S_{8}$ & 115.62 & 0.05 & 116.04 & -0.36 \\
\hline$S_{24}$ & 190.07 & 0.05 & 189.94 & 0.07 & ${ }_{10} S_{9}$ & 110.55 & 0.05 & 110.41 & 0.13 \\
\hline${ }_{g} S_{25}$ & 184.32 & 0.08 & 184.20 & 0.07 & ${ }_{18} S_{11}$ & 103.63 & 0.09 & 103.49 & 0.14 \\
\hline${ }_{3} S_{41}$ & 113.31 & 0.08 & 113.23 & 0.07 & & & & & \\
\hline${ }_{3} S_{42}$ & 111.36 & 0.08 & 111.24 & 0.10 & ${ }_{20} S_{4}$ & 123.18 & 0.05 & 123.15 & 0.02 \\
\hline \multirow[t]{2}{*}{$S_{43}$} & 109.38 & 0.08 & 109.34 & 0.04 & ${ }_{20} S_{0}$ & 102.09 & 0.06 & 101.98 & 0.11 \\
\hline & & & & & ${ }_{21} S_{6}$ & 112.96 & 0.05 & 112.93 & 0.03 \\
\hline \multirow{3}{*}{$\begin{array}{l}{ }_{3} S_{60} \\
{ }_{30} S_{51} \\
{ }_{3} S_{54} \\
S_{50}\end{array}$} & 97.97 & 0.08 & 97.79 & 0.18 & ${ }_{21} S_{\mathrm{g}}$ & 105.36 & 0.05 & 105.18 & 0.17 \\
\hline & 96.44 & 0.07 & 96.36 & 0.08 & ${ }_{22} S_{1}$ & 127.88 & 0.09 & 127.80 & 0.06 \\
\hline & $\begin{array}{l}92.39 \\
87.65\end{array}$ & 0.08 & $\begin{array}{l}92.34 \\
87.55\end{array}$ & 0.05 & & & & & \\
\hline
\end{tabular}

$\begin{array}{lll}S_{58} & 87.65 & 0.05\end{array}$

$\begin{array}{lll}{ }_{3} S_{\text {as }} & 82.38 & 0.13\end{array}$

${ }_{3} S_{70} \quad 76.11$

$\begin{array}{ll}{ }_{9} S_{79} & \mathbf{2 3 . 7 8}\end{array}$

0.13

0.13

${ }_{4} S_{2} \quad 580.81$

$\begin{array}{ll}{ }_{4} S_{3} & 489.05 \\ S_{4} & 439.17\end{array}$

$S_{5} \quad 414.62$

${ }_{4} S_{v} \quad 269.59$

${ }_{4} S_{10} \quad 258.85$

$S_{11} \quad 249.60$

$\begin{array}{ll}S_{12} & 240.78 \\ S_{13} & 232.75\end{array}$

${ }_{4} S_{14} \quad 225.08$

${ }_{4} S_{16} \quad 218.17$

$S_{40} \quad 186.33$

115.44

0.10

0.07
0.1

0.06

0.06

0.08

0.08
0.06

0.06

0.06

0.05

0.06

0.06

${ }_{8} S_{2} \quad 479.34$

${ }_{s} S_{\mathrm{s}} \quad 460.78$

${ }_{5} S_{4} \quad 420.36$

${ }_{6} S_{5} \quad 370.10$

${ }_{5} S_{0} \quad 332.11$

${ }_{{ }^{5} S_{7}} \quad 303.98$

${ }_{8} S_{10} \quad 237.81$

${ }_{8} S_{12} \quad 213.03$

${ }_{8} S_{15} \quad 187.75$

${ }_{5} S_{16} \quad 181.74$

$\begin{array}{ll}{ }_{8} S_{20} & 162.45 \\ { }_{5} S_{25} & 143.59\end{array}$

${ }_{8}{ }_{8} S_{2}$

${ }_{8} S_{36} \quad 116.63$

0.05

0.05

0.05

0.05

0.05
0.05

0.05

0.05

0.05

0.06

0.06

0.05

0.06

0.06

0.05

$\begin{array}{ll}{ }^{\circ} S_{1}^{*} & 505.8 \\ { }^{*} S_{16} & 178.76\end{array}$

${ }^{-} S_{28} \quad 123.51$

$S_{39} \quad 100.68$

${ }_{7} S_{2} \quad 397.37$

${ }_{7} S_{4} \quad 293.20$

${ }_{7} S_{10} \quad 209.42$

${ }_{7} S_{28} \quad 125.48$

${ }_{7} S_{88} \quad 101.74$

${ }_{8} S_{1} \quad 348.12$

$\begin{array}{ll}S_{8} & 239.96 \\ S_{8} S_{90} & 106.04 \\ S_{2} & 310.04\end{array}$
87.55

82.30

76.05

73.68

0.12

0.09

0.08

580.67

488.23

438.48

414.50

269.86

259.01

249.59

241.00

233.00

225.41

218.17

186.17

115.29

477.86

460.63

420.42

370.06

332.29

304.04

283.82

238.02

213.57

188.07

181.92

162.51

143.52

128.52

116.56

504.46

178.59

123.60

100.54

397.07

292.98

209.74

125.79

102.01

347.67

240.20

105.97

309.27
"From this point on, the modes were spot checks and were not used in the inversion.

Dziewonski and Gilbert [1973] and are inconsistent with adjacent modes. When these modes are deleted, $\mathrm{C} 2$ satisfies $68 \%$ of the data to 1 standard deviation and $95 \%$ of the data to 2 standard deviations. Model $\mathrm{C} 2$ is therefore a statistically satisfactory fit to the normal mode data set. The fit to the shortperiod fundamental mode data, ${ }_{0} T_{37}{ }^{0} T_{45}$, is improved when surface wave data are incorporated into the data set.
$-0.06$

0.00

$-0.09$

$-0.11$

-0.15
0.00

0.08

0.13

0.31

0.03

$-0.01$

0.01

$-0.05$

$-0.09$

$-0.09$

$-0.25$

$-0.17$

$-0.10$

-0.04
0.05

$-0.01$

0.06

0.27

0.10

$-0.07$

0.14

0.08

0.08

$-0.15$

$-0.19$

$-0.26$

0.13

$-0.10$

0.07
TABLE 3. Fit of $\mathrm{C} 2$ to Short-Period Toroidal and Love Wave Dispersion Data

\begin{tabular}{|c|c|c|c|c|c|c|}
\hline Mode & $\begin{array}{c}\text { Observa- } \\
\text { tions* }\end{array}$ & Error, \% & $\mathrm{C} 2$ & $\begin{array}{l}\text { C2 Differ- } \\
\text { ence, } \%\end{array}$ & $1066 \mathrm{~B} \dagger$ & $\begin{array}{l}\text { 1066B Dif- } \\
\text { ference, } \%\end{array}$ \\
\hline${ }_{0} T_{21}$ & 345.60 & 0.15 & 345.79 & -0.05 & 346.02 & -0.12 \\
\hline${ }_{0} T_{22}$ & 332.75 & 0.13 & 332.97 & -0.07 & 333.21 & -0.14 \\
\hline${ }_{0} T_{2 \mathrm{a}}$ & 320.92 & 0.12 & 321.10 & -0.06 & 321.35 & -0.14 \\
\hline${ }_{0} T_{24}$ & 310.00 & 0.14 & 310.06 & -0.02 & 310.32 & -0.10 \\
\hline${ }_{0} T_{25}$ & 299.81 & 0.16 & 299.78 & 0.01 & 300.05 & -0.08 \\
\hline${ }_{0} T_{2 \mathrm{~s}}$ & 290.12 & 0.15 & 290.17 & -0.02 & 290.45 & -0.11 \\
\hline${ }_{0} T_{\eta 7}$ & 281.16 & 0.15 & 281.16 & 0.00 & 281.45 & -0.10 \\
\hline${ }_{0} T_{28}$ & 272.70 & 0.15 & 272.70 & 0.00 & 273.00 & -0.11 \\
\hline${ }_{0} T_{20}$ & 264.72 & 0.14 & 264.75 & -0.01 & 265.05 & -0.12 \\
\hline${ }_{0} T_{\mathrm{so}}$ & 257.19 & 0.14 & 257.25 & -0.02 & 257.56 & -0.14 \\
\hline${ }_{0} T_{31}$ & 250.13 & 0.14 & 250.16 & -0.01 & 250.47 & -0.14 \\
\hline${ }_{0} T_{\mathrm{gg}}$ & 243.65 & 0.23 & 243.46 & 0.08 & 243.78 & -0.05 \\
\hline${ }_{0} T_{83}$ & 237.11 & 0.16 & 237.10 & 0.00 & 237.43 & -0.14 \\
\hline${ }_{0} T_{34}$ & 231.06 & 0.17 & 231.07 & -0.00 & 231.40 & -0.15 \\
\hline${ }_{0} T_{s e}$ & 220.07 & 0.26 & 219.89 & 0.08 & 220.22 & -0.07 \\
\hline${ }_{0} T_{87}$ & 214.33 & 0.22 & 214.69 & -0.17 & 215.03 & -0.33 \\
\hline${ }_{0} T_{8 \mathrm{~B}}$ & 209.68 & 0.17 & 209.73 & -0.02 & 210.07 & -0.19 \\
\hline${ }_{0} T_{30}^{\infty}$ & 204.65 & 0.17 & 205.00 & -0.17 & 205.34 & -0.38 \\
\hline${ }_{0} T_{10}$ & 200.19 & 0.17 & 200.48 & -0.15 & 200.82 & -0.32 \\
\hline${ }_{0} T_{41}$ & 195.94 & 0.14 & 196.15 & -0.11 & 196.49 & -0.28 \\
\hline${ }_{0} T_{48}$ & 191.65 & 0.19 & 192.00 & -0.18 & 192.34 & -0.36 \\
\hline${ }_{0} T_{48}$ & 187.73 & 0.19 & 188.02 & -0.15 & 188.36 & -0.34 \\
\hline${ }_{0} T_{\mu}$ & 183.99 & 0.17 & 184.21 & -0.12 & 184.55 & -0.30 \\
\hline${ }_{0} T_{16}$ & 180.38 & 0.15 & 180.54 & -0.09 & 180.88 & -0.28 \\
\hline${ }_{0} T_{40}$ & 176.91 & 0.15 & 177.02 & -0.06 & 177.36 & -0.25 \\
\hline
\end{tabular}

"Average of Kanamori [1970], Dziewonski et al. [1972], and Gilbert and Dziewonski [1975].

tGilbert and Dziewonski [1975]. 
TABLE 4. Group Velocities

\begin{tabular}{|c|c|c|c|c|}
\hline \multirow[b]{2}{*}{ Mode } & \multicolumn{2}{|c|}{$T, \mathrm{~s}$} & \multicolumn{2}{|c|}{$U, \mathrm{~km} / \mathrm{s}$} \\
\hline & DMB & $\mathrm{C} 2$ & DMB & $\mathrm{C} 2$ \\
\hline${ }_{0} S_{10}$ & 579.40 & 579.32 & 5.67 & 5.66 \\
\hline$S_{18}$ & 502.43 & 502.45 & 5.01 & 5.01 \\
\hline${ }_{0} S_{18}$ & 426.12 & 426.11 & 4.54 & 4.55 \\
\hline$S_{21}$ & 335.93 & 335.68 & 3.93 & 3.94 \\
\hline$\omega_{25}$ & 297.78 & 297.57 & 3.73 & 3.72 \\
\hline$S_{39}$ & 268.48 & 268.36 & 3.62 & 3.62 \\
\hline$S_{36}$ & 234.58 & 234.52 & 3.57 & 3.58 \\
\hline$S_{40}$ & 212.34 & 212.25 & 3.58 & 3.59 \\
\hline$S_{46}$ & 193.88 & 193.74 & 3.60 & 3.62 \\
\hline${ }_{0} T_{10}$ & 617.47 & 619.03 & 5.07 & 5.01 \\
\hline${ }_{0} T_{18}$ & 503.38 & 505.16 & 4.76 & 4.74 \\
\hline$T_{18}$ & 428.14 & 429.52 & 4.58 & 4.58 \\
\hline${ }_{0} T_{21}$ & 344.90 & 345.79 & 4.46 & 4.46 \\
\hline${ }_{0} T_{25}$ & 299.12 & 299.78 & 4.43 & 4.43 \\
\hline${ }_{0} T_{2 \mathrm{~g}}$ & 264.19 & 264.75 & 4.42 & 4.41 \\
\hline${ }_{0} T_{41}$ & 195.68 & 196.15 & 4.42 & 4.41 \\
\hline${ }_{0} T_{46}$ & 176.62 & 177.02 & 4.42 & 4.41 \\
\hline
\end{tabular}

DMB denotes Dziewonski et al. [1972].

There is considerable spread in measured values for the shorter-period fundamental toroidal oscillations. This probably represents real lateral variations in the structure of the upper mantle. Kanamori [1970] and Dziewonski et al. [1972] have measured the dispersion of Love waves over a considerable number of great circle paths. These data can be used to augment the data of Gilbert and Dziewonski [1975] in order to obtain a more representative gross earth data set. Table 3 gives the values obtained for ${ }_{0} T_{21}-0 T_{48}$ by averaging the above data sets with equal weight. The error is the standard deviation of the data groups and does not include the errors associated with the individual groups. Table 2 also gives some spot checks of the very high spheroidal overtone data (37 modes). These additional modes were not used in the inversion, but the fit is comparable to that of the models of Gilbert and Dziewonski [1975].

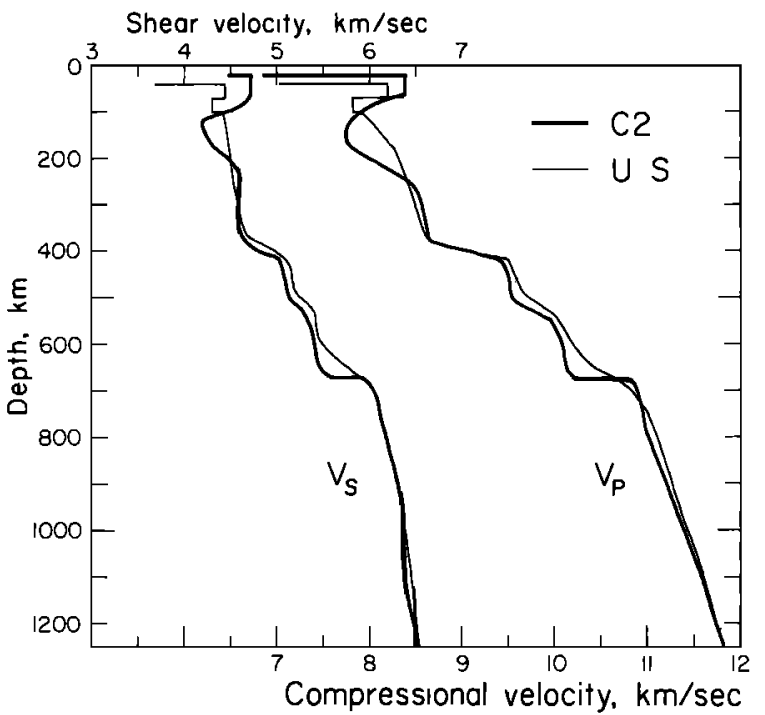

Fig. 2. Upper mantle structure of $\mathrm{C} 2$ compared with results of Helmberger and Wiggins [1971] and Helmberger and Engen [1974] which are based on amplitude and wave form studies in the western United States.

C2 group velocities are compared with the results of Dziewonski et al. [1972] in Table 4. The data set is not so large or representative in this case, but the agreement is good.

Although the number of modes inverted is considerably less than the 1066 considered by Gilbert and Dziewonski [1975], they constitute a representative data set, particularly when one considers that the total data set includes only 57 significant earth data [Backus and Gilbert, 1968; Gilbert et al., 1973; Gilbert and Dziewonski, 1975]. Many of the additional modes do not contain information independent of that contained in the differential travel times and the modes considered in this paper. The additional modes also do not contribute substantially to the resolving power required to distinguish between models of the upper mantle. For example, compare the upper mantles of 1066A and 1066B in Gilbert and Dziewonski [1975]. The former used a smooth upper mantle as a starting

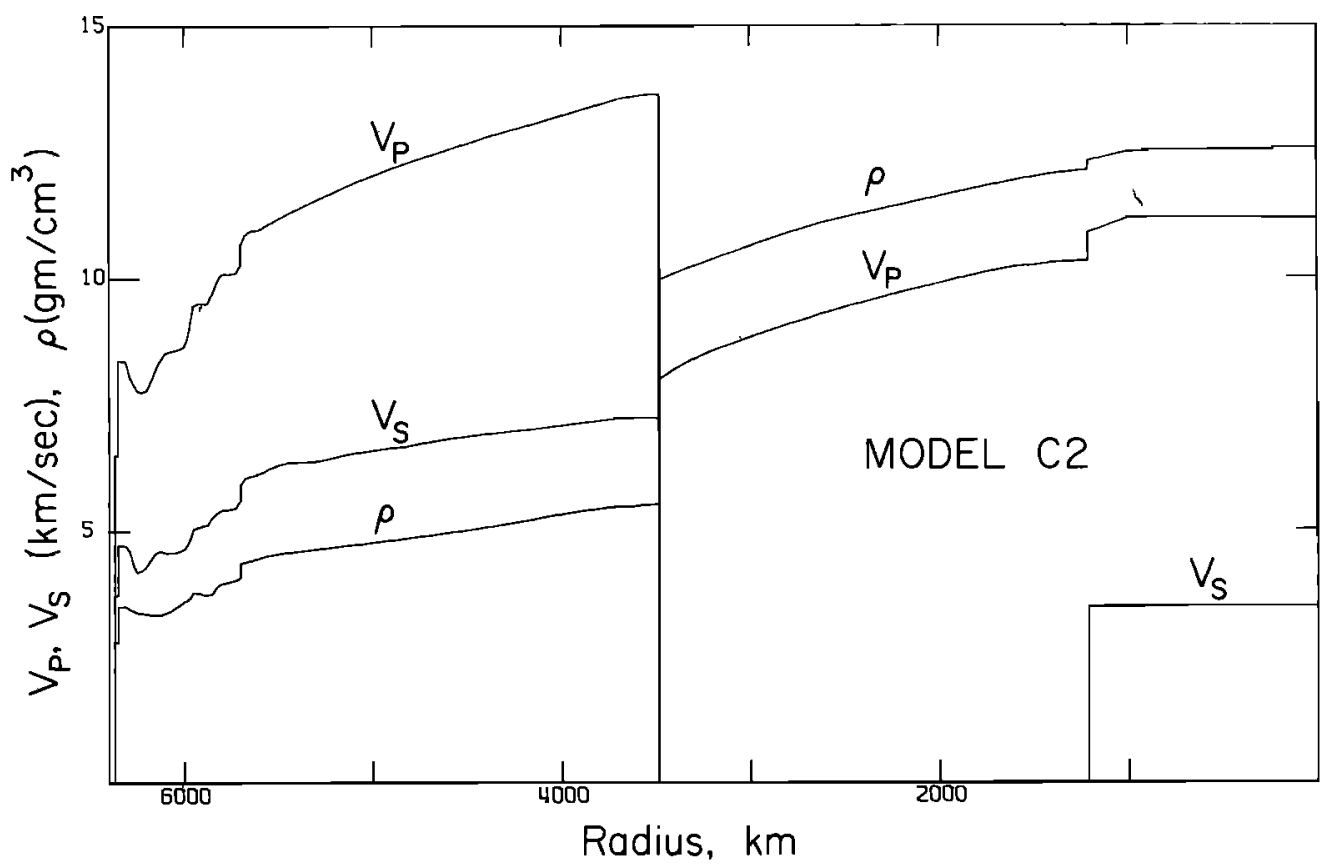

Fig. 1. Model C2. $V_{p}$ (compressional velocity), $V_{s}$ (shear velocity), and $\rho$ (density) as a function of radius. 


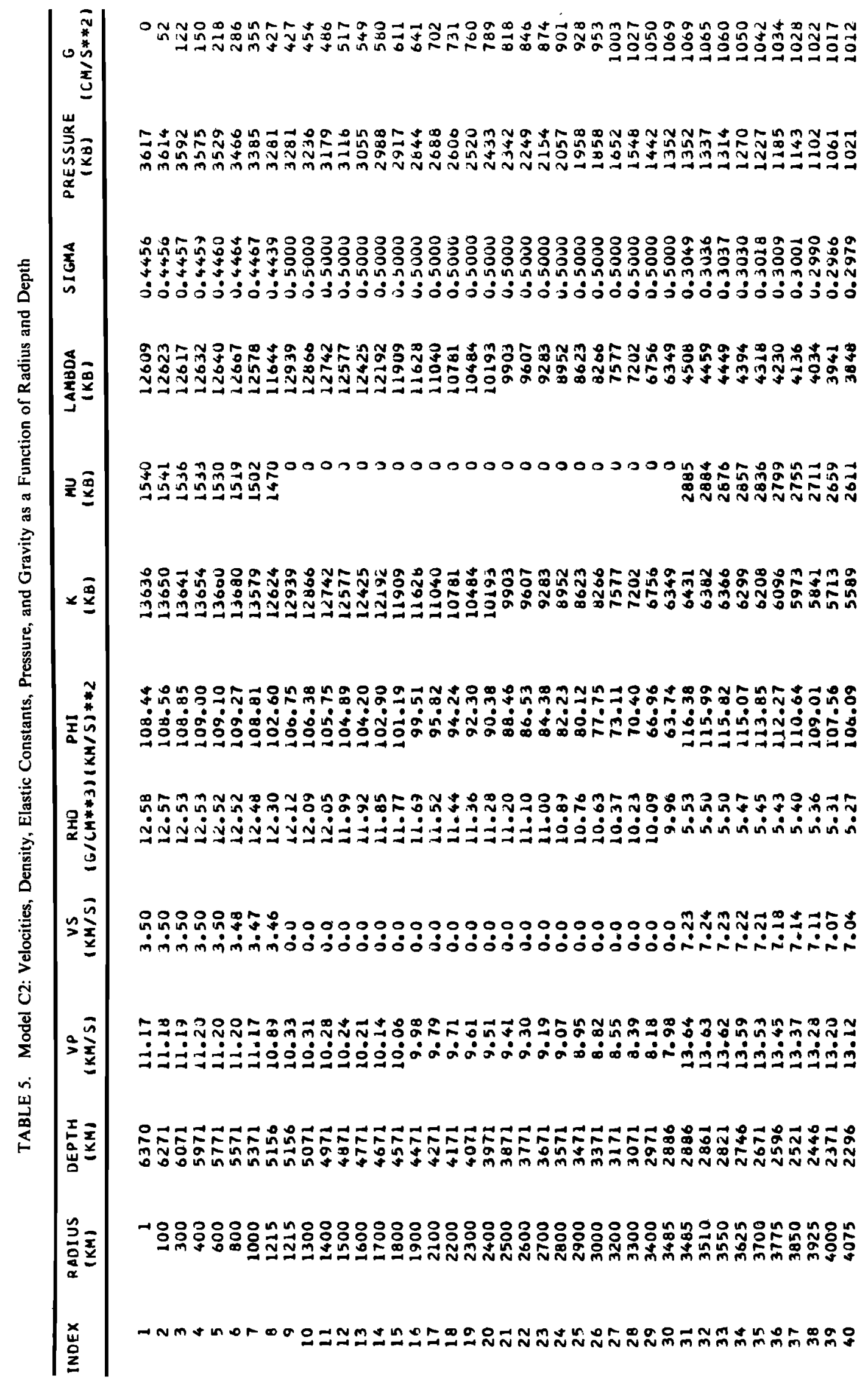




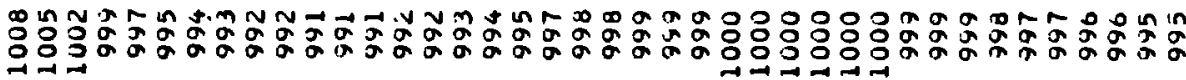

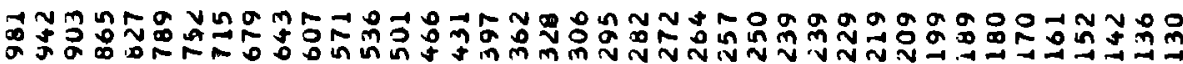

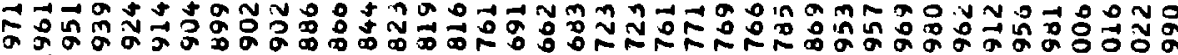

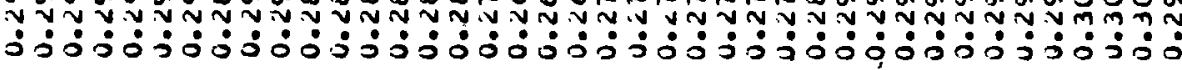

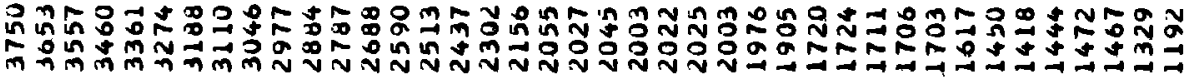

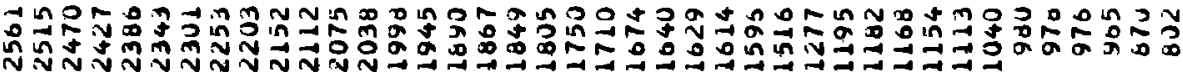

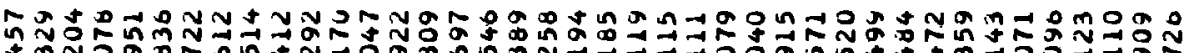

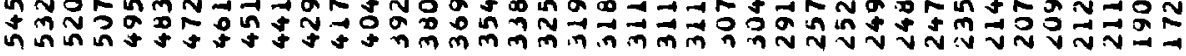

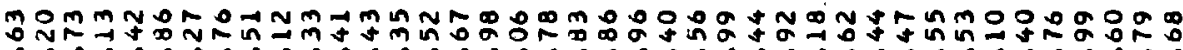

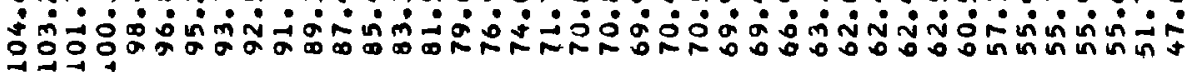

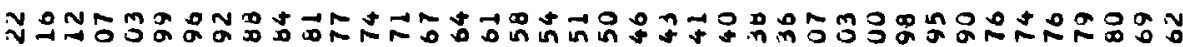

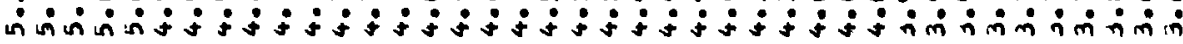

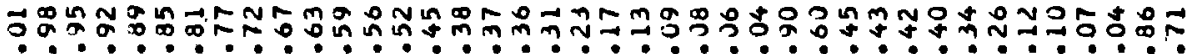

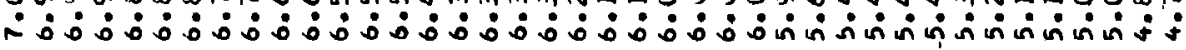

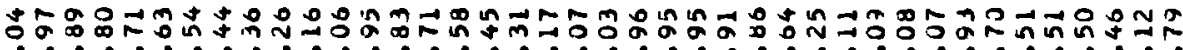

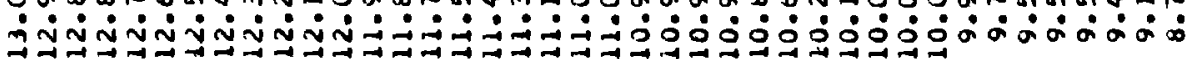

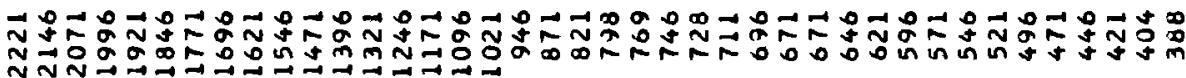

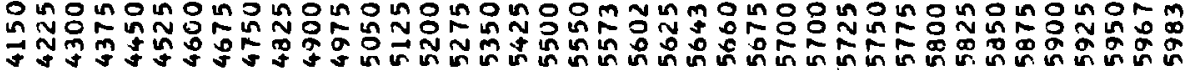

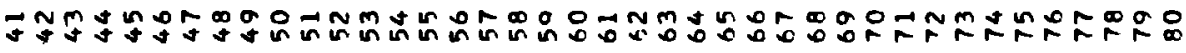




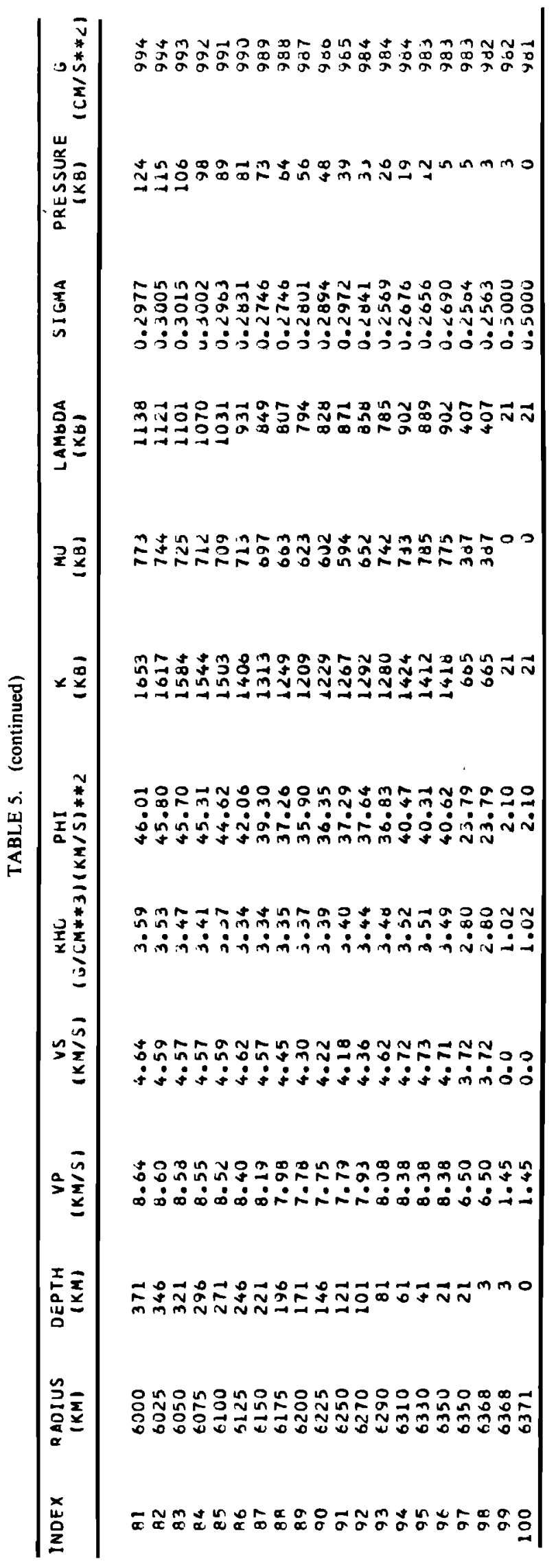

model, and the latter used BI, a model with two upper mantle discontinuities, as a starting model. The smooth starting model remained smooth, showing that the additional modes cannot resolve the detail which is apparent from body wave studies. Additionally, when B1 was subjected to reinversion with the use of all 1066 modes, there were very few changes required, usually amounting to less than $0.05 \%$, and the changes introduced in the upper mantle were in the same direction and generally of the same nature as the differences between $\mathrm{C} 2$ and $\mathrm{B} 1$. We feel therefore that our procedure of using high-resolution body wave structures as starting models in the inversion and checking the resulting model against both the very high overtone data and the body wave data is at least equivalent to, and perhaps better than, a procedure that relies exclusively on the short-period higher-mode data. The fact that the lower mantle and core of $C 2$ are very similar to those of the Gilbert-Dziewonski models, which were based on all 1066 modes, justifies this approach.

\section{The Resulting Model}

The inverted model, $\mathrm{C} 2$, is shown in Figures 1 and 2. The model parameters are given in Table 5 . In addition to $V_{p}, V_{s}$, and density as a function of layer index, radius, and depth we also tabulate the seismic parameter $\Phi\left(=K / \rho=V_{p}^{2}-\right.$ $\left.(4 / 3) V_{s}^{2}\right)$, bulk modulus $K$, rigidity $\mu$, Lamé constant $\lambda$, Poisson's ratio $\sigma$, pressure, and gravity. Also shown in Figure 2 are the Helmberger-Wiggins-Engen profiles, which can be considered models of the upper mantle under western North America. Except for the large differences in the structure of the lowvelocity zone and the lithospheric lid the main effect of the inversion was to decrease both $P$ and $S$ velocities between the $400-$ and $670-\mathrm{km}$ discontinuities by about $0.05-0.1 \mathrm{~km} / \mathrm{s}$.

The average lithospheric velocities of $\mathrm{C} 2$ are 8.38 and 4.71 $\mathrm{km} / \mathrm{s}$ for $V_{p}$ and $V_{s}$, respectively. These can be compared with $8.28 \pm 0.03$ and $4.79 \pm 0.04 \mathrm{~km} / \mathrm{s}$ recorded over long distances in the Pacific [Sutton and Walker, 1972] and 8.27 \pm 0.01 and $4.75 \pm 0.07 \mathrm{~km} / \mathrm{s}$ for $P_{n}$ and $S_{n}$ over the Australian shield [Simpson, 1973]. Hart and Press [1973] determined a value of $4.71 \mathrm{~km} / \mathrm{s}$ for $S_{n}$ for 50- to 150-m.y.-old oceanic lithosphere. There is evidence from refraction studies that $V_{p}$ may be as high as $8.6 \mathrm{~km} / \mathrm{s}$ in the lower lithosphere [e.g., Kosminskaya et al. 1972]. These studies are consistent with the average velocities of the lithosphere found here. The depth to the top of the low-velocity zone is $61 \mathrm{~km}$, although this could be increased to about $80 \mathrm{~km}$ if the entry into the low-velocity zone is abrupt rather than gradual. The thickness of the LVZ is about 180 $\mathrm{km}$. The density of the uppermost mantle is $3.50 \mathrm{~g} / \mathrm{cm}^{3}$ (see, however, the discussion on resolving power below). A small amount of structure in the shear velocity is evident between about $670-$ and $1200-\mathrm{km}$ depth. This results in a pronounced dip in the $S$ wave residual near $40^{\circ}$, as required by the studies of Ibrahim and Nuttli [1967] and Hart [1975]. The shape of the $S$ wave residual curve beyond $60^{\circ}$ for $\mathrm{C} 2$ is also more in line with body wave studies [i.e., Hales and Roberts, 1970] than is that for B1.

The major effect of the inversion on core velocities is an increase of about $0.05 \mathrm{~km} / \mathrm{s}$ from the starting model. The other effects of the inversion are slight changes in the velocity gradient in the outer $400 \mathrm{~km}$ of the core, an increase in the velocity gradient in the outer part of the inner core, and a decrease in the velocity jump across the outer core-inner core boundary. The density jump and compressional velocity jump at the boundary are, $0.02 \mathrm{~g} / \mathrm{cm}^{3}$ and $0.56 \mathrm{~km} / \mathrm{s}$, respectively. The average density, compressional velocity, and shear velocity of the inner core are $12.52 \mathrm{~g} / \mathrm{cm}^{3}, 11.19 \mathrm{~km} / \mathrm{s}$, and 3.50 
$\mathrm{km} / \mathrm{s}$. The shear velocity at the top of the inner core is 3.46 $\mathrm{km} / \mathrm{s}$.

The small compressional velocity jump at the inner core-outer core boundary $(+0.56 \mathrm{~km} / \mathrm{s})$ is in agreement with the evidence from amplitudes of long-period core phases [Müler, 1973] which gives $0.58 \mathrm{~km} / \mathrm{s}$. The high velocity gradient at the top of the inner core is also consistent with amplitude studies [Müller, 1973]. The shear velocity at the top of the inner core, $3.46 \mathrm{~km} / \mathrm{s}$, is in general agreement with the bounds, 3-4 km/s, established by Müller [1973].

There is some evidence for inhomogeneity in the outer core, at both its upper and its lower boundaries. The velocity gradient is about $0.24 \mathrm{~km} / \mathrm{s}$ per $100 \mathrm{~km}$ at the top of the core decreasing to $0.13 \mathrm{~km} / \mathrm{s}$ at a radius of $2800 \mathrm{~km}$ or about 700 $\mathrm{km}$ deep into the core. The gradient then decreases gradually to $0.08 \mathrm{~km} / \mathrm{s}$ per $100 \mathrm{~km}$ at a radius of $1700 \mathrm{~km}$. The velocity increases much more slowly, $0.03 \mathrm{~km} / \mathrm{s}$ per $100 \mathrm{~km}$ in the lowermost $500 \mathrm{~km}$ of the outer core. A similar effect occurs in the density profile, with a relatively high density gradient in the outer portion of the core compared with that at deeper levels.

It is of interest to compare the lower mantle and core of $\mathrm{C} 2$ with 1066A and 1066B [Gilbert and Dziewonski, 1975]. Gilbert and Dziewonski [1975] utilized the complete high-overtone data set, while we leaned more heavily on the nominally equivalent body waves and the more abundant fundamental and lower-overtone data and only utilized a sparse sampling of the high-overtone data. Below a radius of $5600 \mathrm{~km}$ the mantle shear velocities and densities for these models are virtually identical. The $P$ velocities differ at most by $0.2 \mathrm{~km} / \mathrm{s}$; the main difference is that the $P$ velocity for the 1066 models has a long wavelength oscillation, while that of the $\mathrm{C} 2$ is much smoother. Dziewonski et al. [1975], using the full mode data set, also have a smooth lower mantle for $V_{p}$. The density and $V_{p}$ in the core are also in very good agreement. There are small differences in the inner core for $V_{p}$ and $V_{s}$. In $\mathrm{C} 2$ the slight structure for $V_{p}$ in the inner core, particularly the rapid increase in the outer portion, is inherited from the starting model of Whitcomb [1973] and is therefore a requirement of the core phases rather than of the modes. The differences between $\mathrm{C2}, 1066 \mathrm{~A}$, and $1066 \mathrm{~B}$ in the inner core are probably unresolvable by using the modes alone. The differences are slight. For example, $V_{p}$ at the top of the inner core ranges from $10.97 \mathrm{~km} / \mathrm{s}(1066 \mathrm{~A}), 11.04$ $\mathrm{km} / \mathrm{s}(1066 \mathrm{~B})$, and $10.89 \mathrm{~km} / \mathrm{s}(\mathrm{C} 2)$, a spread of $1 \%$. The central $V_{p}$ is $11.34 \mathrm{~km} / \mathrm{s}(1066 \mathrm{~A}), 11.28 \mathrm{~km} / \mathrm{s}(1066 \mathrm{~B})$, and $11.17 \mathrm{~km} / \mathrm{s}(\mathrm{C} 2)$, also a spread of $1 \%$. The average $V_{s}$ for the inner core is $3.57 \mathrm{~km} / \mathrm{s}(1066 \mathrm{~A}), 3.50 \mathrm{~km} / \mathrm{s}(1066 \mathrm{~B})$, and 3.48 $\mathrm{km} / \mathrm{s}(\mathrm{C2})$. The major difference among the models is the density of the inner core. This is not unexpected, since the resolving power for density is very poor in this region. This is unfortunate, since the density is the main constraint on the composition of the inner core. If the density jump at the outer core-inner core boundary is small, as in $\mathrm{C} 2$, then the inner core can be the same material as the outer core, since freezing at core pressures can be expected to increase the density only slightly. If the density jump is large, then it is probable that the inner core is lacking in the light elements that are required to satisfy the outer core densities. Average inner core densities are $13.12 \mathrm{~g} / \mathrm{cm}^{3}(1066 \mathrm{~A}), 12.85 \mathrm{~g} / \mathrm{cm}^{3}(1066 \mathrm{~B})$, and 12.35 $\mathrm{g} / \mathrm{cm}^{\mathrm{a}}(\mathrm{C} 2)$. The density of iron at inner core pressures is about $12.9-13.4 \mathrm{~g} / \mathrm{cm}^{3}$.

\section{RESOLUTION}

The resolving power of gross earth data has been discussed by Backus and Gilbert [1968] and Jordan and Anderson [1974]. Although the data set used in the present inversion is more extensive than that used by the latter authors, we use their estimates of averaging lengths as conservative guides. The trade-offs between parameters such as density and shear velocity are also discussed by Jordan and Anderson [1974], and Dziewonski [1971]. These trade-offs make it particularly important to have independent estimates of the shear velocity structure and to fit first those modes that are sensitive to shear velocity.

Resolution is poor for density below $2400 \mathrm{~km}$, shear velocity structure in the inner core and in the lower $500 \mathrm{~km}$ of the mantle, and compressional velocity in the vicinity of $2400-\mathrm{km}$ radius. In these regions, only very long wavelength perturbations from the starting model are justified by the data. The averaging lengths for shear velocity in the upper mantle and transition region are about 200 and $400 \mathrm{~km}$, respectively. The averaging kernels for $V_{p}$ in the outer core are about 1000 km. As Jordan and Anderson [1974] point out, the density of the lithosphere cannot be discussed with any useful precision because the averaging length for density in the upper mantle is about $400 \mathrm{~km}$. However, the high average shear velocity in the lithosphere is resolvable and is consistent with body wave data. Structure in the lithosphere is not resolvable. The averaging lengths for density in the lower mantle are about 1000 $\mathrm{km}$.

Considering the above facts, the slight reversals in shear velocity below $246-\mathrm{km}(0.05 \mathrm{~km} / \mathrm{s}$ over $100 \mathrm{~km})$ depth and in density below $421-\mathrm{km}\left(0.06 \mathrm{~g} / \mathrm{cm}^{3}\right.$ over $\left.25 \mathrm{~km}\right)$ depth are clearly not resolvable.

\section{Compressional Waves}

Most recent studies indicate that the JB tables for $\boldsymbol{P}$ waves are slow by up to $3 \mathrm{~s}$. Qualitatively, the present study indicates the same thing, but the average discrepancy between $30^{\circ}$ and $95^{\circ}$ is only $1.2 \mathrm{~s}$ with maximum deviations from JB times near $30^{\circ}(1.6 \mathrm{~s})$ and between $55^{\circ}$ and $75^{\circ}(1.7-3.0 \mathrm{~s})$. Model $\mathrm{C} 2$ is $1.5 \mathrm{~s}$ slow, on the average, over the range $30^{\circ}-95^{\circ}$, in comparison with the 1968 tables, with the residuals decreasing from $2.4 \mathrm{~s}$ at $30^{\circ}$ to $0.8 \mathrm{~s}$ at $80^{\circ}$ and increasing to $1.5 \mathrm{~s}$ at $95^{\circ}$. A possible bias of this type in the 1968 tables was pointed out by Jordan and Anderson [1974]. The travel times of Hales et al. [1968] agree with those predicted by $C 2$ to within $0.6 \mathrm{~s}$ with maximum deviations of $1 \mathrm{~s}$ at $45^{\circ}$ and $90^{\circ}$. Model C2 averages 0.6 slower than the data of Hales et al. [1968]. The discrepancies between the various body wave studies confound efforts to determine differences between the 'average' mantle (free oscillations) and tectonic to continental paths (most body wave studies), but the present study combined with the most recent body wave data suggests that the average earth is about $0.6 \mathrm{~s}$ slower than that portion of the earth available to study by body wave techniques, i.e., continental sources and receivers. Alternatively, one could say that $\mathrm{C} 2$ is consistent with $P$ wave travel time studies, since it falls between the JB and the 1968 solutions [Herrin et al., 1968] and is close to solutions of Cleary and Hales [1966], Hales et al. [1968], and Carder et al. [1966]. Throughout most of the distance range between $30^{\circ}$ and $95^{\circ}, \mathrm{C} 2$ is slightly slower than the three 1966 studies and is closest to Cleary and Hales [1966]; see Figure 3 and Table 6.

Table 7 compares the apparent velocities $(d t / d \Delta)$ of $\mathrm{C} 2$ with four sets of published data. The fit is satisfactory in that predicted values fall within the scatter of the observations except near $85^{\circ}$, but even there the difference is only $0.6 \%$.

Model C2 averages $2 \mathrm{~s}$ faster than JB times for $P c P$ between $30^{\circ}$ and $90^{\circ}$ (Table 8 ). The difference in the size of the core accounts for about $1.8 \mathrm{~s}$ of this difference. The remainder is accounted for by the 0.3-s difference in travel times between $\mathrm{JB}$ 


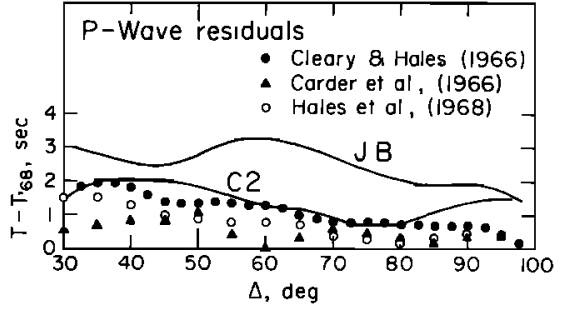

Fig. 3. Compressional velocity residuals, relative to the 1968 tables [Herrin et al., 1968], of C2 and other recent studies. Jeffreys-Bullen (JB) times are also shown.

and $C 2$ at $95^{\circ} . P c P$ times from Pacific events [Gogna, 1973] agree with $C 2$ to $0.3 \mathrm{~s}$, ranging from $+2.2 \mathrm{~s}$ at $50^{\circ}$ to $-2.0 \mathrm{~s}$ at $80^{\circ}$ (observed minus computed values). The modified $P c P$ times [Engdahl and Johnson, 1974] consistent with the 1968 tables average $1.3 \mathrm{~s}$ faster than model $\mathrm{C} 2$. Since these times were determined from differential $P C P$-P times and the B1, and $C 2$, core radius, this difference must be accounted for in mantle velocities. In fact, the 1968 tables average $1.5 \mathrm{~s}$ faster than $\mathrm{C} 2$ for $P$ waves between $30^{\circ}$ and $95^{\circ}$. Within the uncertainty of the data, no statement can be made from $P c P$ data regarding the differences between the average earth and the body wave solutions. The $C 2 P c P-P$ times (Table 9) average $0.5 \mathrm{~s}$ fast between $30^{\circ}$ and $60^{\circ}$ and $0.3 \mathrm{~s}$ slow beyond $65^{\circ}$ but seem to be generally consistent with the data.

The core phase $P K P$ averages $1.7 \mathrm{~s}$ faster for $\mathrm{C} 2$ than for JB. This is in agreement, within $0.3 \mathrm{~s}$, with the differences in $P c P$ times and therefore can be accounted for by differences in core radii and mantle velocities. The differential core times $\left(\boldsymbol{P}_{\mathrm{AB}}{ }^{\prime}-\right.$ $\left.P_{\mathrm{DF}}{ }^{\prime}, P_{\mathrm{BC}}{ }^{\prime}-P_{\mathrm{DF}}{ }^{\prime}\right)$ agree with the recent study of Whitcomb [1973], with differences ranging from +0.4 to $-0.9 \mathrm{~s}$. For comparison, other PKP data are tabulated in Table 10. The average difference between $C 2$ and the 1968 tables is $-0.3 \mathrm{~s}$. The $P K P$ times for the $\mathrm{AB}$ and $\mathrm{BC}$ branches for $\mathrm{C} 2$ are generally bracketed by the values given in the 1968 tables and the times given by Whitcomb [1973]. However, the DF branch is generally $1-2 \mathrm{~s}$ fast. This could be corrected (1) by decreasing the velocity of the region surrounding the inner core, keeping the velocity jump at the inner core fixed or increasing it at most by $0.14 \mathrm{~km} / \mathrm{s}$ in order to satisfy the amplitude data, (2) by decreasing the radius of the inner core, or (3) by decreasing the

TABLE 6. Compressional Wave Travel Times and Errors

\begin{tabular}{lccccccc}
\hline & & & & \multicolumn{3}{c}{ Difference } \\
\cline { 5 - 8 }$\Delta$, deg & JB & HCR & 1968 & C2 & JB & HCR & 1968 \\
\hline 30 & 372.5 & 371.0 & 368.5 & 370.9 & 1.6 & 0.1 & -2.4 \\
35 & 416.1 & 414.8 & 413.3 & 415.3 & 0.8 & -0.5 & -2.0 \\
40 & 458.1 & 457.0 & 455.7 & 457.8 & 0.3 & -0.8 & -2.1 \\
45 & 498.9 & 497.4 & 496.4 & 498.4 & 0.5 & -1.0 & -2.0 \\
50 & 538.0 & 536.1 & 535.2 & 537.0 & 1.0 & -0.9 & -1.8 \\
55 & 575.4 & 573.0 & 572.2 & 573.7 & 1.7 & -0.7 & -1.5 \\
60 & 610.7 & 608.2 & 607.4 & 608.7 & 2.0 & -0.5 & -1.3 \\
65 & 644.0 & 641.6 & 640.9 & 642.1 & 1.9 & -0.5 & -1.2 \\
70 & 675.4 & 673.1 & 672.7 & 673.6 & 1.8 & -0.5 & -1.4 \\
75 & 705.0 & 702.9 & 702.6 & 703.3 & 1.7 & -0.4 & -0.7 \\
80 & 732.7 & 730.8 & 730.6 & 731.4 & 1.3 & -0.6 & -0.8 \\
85 & 758.5 & 756.9 & 756.6 & 757.7 & 0.8 & -0.8 & -1.1 \\
90 & 782.7 & 781.1 & 780.7 & 782.1 & 0.6 & -1.0 & -1.4 \\
95 & 805.7 & & 803.9 & 805.4 & 0.3 & & -1.5 \\
Average & & & & & & \\
\multicolumn{2}{c}{ difference } & & & & +1.2 & -0.6 & -1.5 \\
\hline
\end{tabular}

Travel times are in seconds. JB denotes Jeffreys and Bullen [1940]; HCR, Hales et al. [1968]; and 1968, Herrin et al. [1968].
TABLE 7. Observed and Computed $d t / d \Delta$ of $\mathrm{P}$ Waves

\begin{tabular}{cccccc}
\hline$\Delta$, deg & HCR & CGJ & LJ & DJC* & C2 \\
\hline 30 & 8.94 & 8.88 & 8.92 & $9.13 \pm 0.05$ & 8.99 \\
35 & 8.60 & 8.67 & 8.60 & $8.70 \pm 0.05$ & 8.67 \\
40 & 8.26 & 8.30 & 8.38 & $8.26 \pm 0.07$ & 8.32 \\
45 & 7.91 & 7.99 & 7.90 & $8.11 \pm 0.10$ & 7.93 \\
50 & 7.56 & 7.52 & 7.51 & $7.52 \pm 0.10$ & 7.53 \\
55 & 7.21 & 7.10 & 7.22 & $7.19 \pm 0.08$ & 7.17 \\
60 & 6.86 & 6.84 & 6.75 & $6.95 \pm 0.07$ & 6.83 \\
65 & 6.50 & 6.66 & 6.53 & $6.69 \pm 0.08$ & 6.49 \\
70 & 6.14 & 6.17 & 6.24 & $6.21 \pm 0.09$ & 6.13 \\
75 & 5.77 & 5.77 & 5.83 & $5.88 \pm 0.06$ & 5.78 \\
80 & 5.40 & 5.35 & 5.48 & $5.47 \pm 0.06$ & 5.44 \\
85 & 5.03 & 4.98 & 4.93 & $4.95 \pm 0.06$ & 5.06 \\
90 & 4.66 & 4.74 & 4.65 & $4.60 \pm 0.09$ & 4.75 \\
95 & 4.28 & 4.55 & 4.48 & $4.52 \pm 0.07$ & 4.57 \\
\hline
\end{tabular}

Values for $d t / d \Delta$ are in seconds per degree. HCR denotes Hales et al. [1968]; CGJ, Carder et al. [1966]; LJ, Johnson [1969]; and DJC, Corbishley [1970].

*Uncertainty is $95 \%$ confidence interval.

average velocity in the inner core by $0.5-0.1 \mathrm{~km} / \mathrm{s}$, again honoring the velocity jump at the boundary. Only the last alternative would be consistent with the $P K i K P-P c P$ data which, as they stand, suggest the reverse of options (1) and (2).

The differential time $P K i K P$ - $P c P$ (Table 11 ) is a measure of the radius of the inner core. Model $\mathrm{C} 2$ averages $0.6 \mathrm{~s}$ slower than the data of Engdahl et al. [1974]. On the assumption that core velocities in $\mathrm{C} 2$ are accurate this suggests that the inner core is $3 \mathrm{~km}$ larger than $\mathrm{C} 2$, or $1218 \mathrm{~km}$. The scatter in the data, however, is such $(-1.4$ to $+0.2 \mathrm{~s})$ that inner core radii from 1214 to $1222 \mathrm{~km}$ are acceptable. The uncertainty in $P c P$ and $P K P$, i.e., average velocities in the mantle, core, and outer core radius, is such that the value $1227.4 \pm 0.6 \mathrm{~km}$, preferred by Engdahl et al. [1974], is an acceptable solution, although their error estimate appears to be optimistic. An uncertainty in outer core travel times of $1 \mathrm{~s}$ immediately introduces an error of $5 \mathrm{~km}$ in the radius of the inner core.

\section{SHEAR Waves}

The scatter in shear wave travel times is well known. Part of the difficulty is related to the fact that shear waves are not first arrivals but must be picked out of the $P$ coda; transformation

TABLE 8. PcP Times and Errors

\begin{tabular}{|c|c|c|c|c|c|c|c|}
\hline \multirow[b]{2}{*}{$\Delta, \operatorname{deg}$} & \multirow[b]{2}{*}{ JB } & \multirow[b]{2}{*}{ Gogna } & \multirow[b]{2}{*}{$68 \mathrm{M}$} & \multirow[b]{2}{*}{ C2 } & \multicolumn{3}{|c|}{ Difference } \\
\hline & & & & & JB & Gogna & $68 \mathrm{M}$ \\
\hline 30 & 554.9 & 553.0 & 551.1 & 552.2 & 2.7 & 0.8 & -1.1 \\
\hline 35 & 568.6 & 567.4 & 564.9 & 566.1 & 2.5 & 1.3 & -1.2 \\
\hline 40 & 583.9 & 583.2 & 580.3 & 581.4 & 2.5 & 1.8 & -1.1 \\
\hline 45 & 600.5 & 600.2 & 596.9 & 598.1 & 2.4 & 2.1 & -1.2 \\
\hline 50 & 618.3 & 618.2 & 614.8 & 616.0 & 2.3 & 2.2 & -1.2 \\
\hline 55 & 637.0 & 636.8 & 633.7 & 634.9 & 2.1 & 1.9 & -1.2 \\
\hline 60 & 656.6 & 656.0 & 653.3 & 654.6 & 2.0 & 1.4 & -1.3 \\
\hline 65 & 676.9 & 675.9 & 673.7 & 675.0 & 1.9 & 0.9 & -1.3 \\
\hline 70 & 697.8 & 695.6 & 694.7 & 696.0 & 1.8 & -0.4 & -1.3 \\
\hline 75 & 719.1 & 716.0 & 716.1 & 717.4 & 1.7 & -1.4 & -1.3 \\
\hline 80 & 740.6 & 737.1 & 737.8 & 739.1 & 1.5 & -2.0 & -1.3 \\
\hline 85 & 762.3 & 759.2 & 759.7 & 761.1 & 1.2 & -1.9 & -1.4 \\
\hline 90 & 784.2 & 781.6 & 781.8 & 783.3 & 0.9 & -1.7 & -1.5 \\
\hline $\begin{array}{r}\text { Avera } \\
\text { diff }\end{array}$ & & & & & +2.0 & +0.3 & -1.3 \\
\hline
\end{tabular}

Values are in seconds. JB denotes Jeffreys and Bullen [1940]; Gogna, Gogna [1973]; and 68M, Engdahl and Johnson [1974]. 


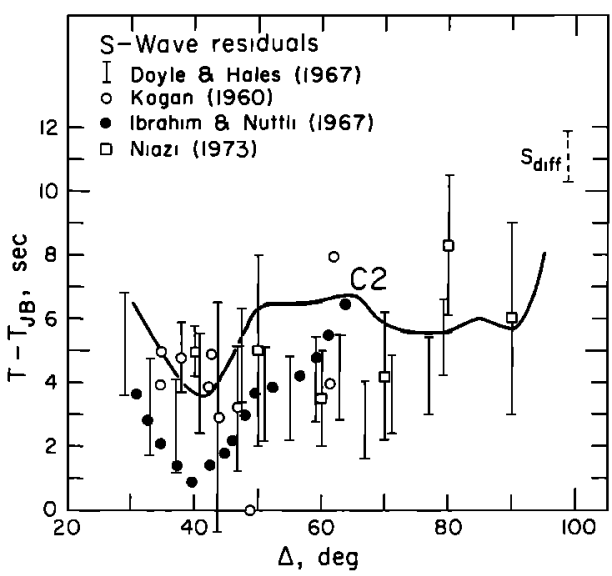

Fig. 4. Shear velocity residual, relative to the Jeffreys-Bullen (JB) tables, of $\mathrm{C} 2$ and other recent studies.

of $S$ to $P$ at upper mantle and crustal discontinuities can also bias ' $S$ readings' toward earlier arrivals. Shear waves are much longer in period than $\boldsymbol{P}$ waves and suffer more attenuation. They are also not efficiently generated by underground explosions. All of the above facts combine to make shear wave arrival times at least 4 times more uncertain than $P$ wave arrival times. In addition, there seem to be real lateral variation effects, including source, path, and receiver variations, that are more pronounced for $S$ waves than for $P$ waves. Additional complications include large-amplitude surface reflections and interference by $P L$ waves [Helmberger and Engen. 1974].

In comparison with published $S$ wave travel times (Table 12), C2 is 4.4-5.9 s slow between $30^{\circ}$ and $95^{\circ}$. In comparison with unpublished data of F. E. Followill and O. Nuttli (1971), appropriate for paths to the western United States (tectonic), $\mathrm{C} 2$ is, on the average, $0.5 \mathrm{~s}$ slow. For other paths the discrepancy varies from about $5 \mathrm{~s}$ at $35^{\circ}$ to $3 \mathrm{~s}$ at $95^{\circ}$. From about $30^{\circ}-40^{\circ}, \mathrm{C} 2$ agrees with data of Kogan [1960] for Pacific surface explosions and falls between 'continental' and 'tectonic' solutions. Beyond $40^{\circ}, \mathrm{C} 2$ is $2-4 \mathrm{~s}$ slow in comparison with most shear wave travel times. Some of the data reported by Kogan [1960], Niazi [1973], and Bolt et al. [1970] are even slower than $\mathrm{C} 2$. However, studying the data, one gets the impression that $\mathrm{C} 2$ is slow by $2-4 \mathrm{~s}$ in comparison with the majority of shear wave travel time studies.

Between $30^{\circ}$ and $45^{\circ}$ and $75^{\circ}$ and $80^{\circ}$ (Table 12) the shear

TABLE 9. $P c P-P$ Times (Surface Focus) and Errors

\begin{tabular}{crrrr}
\hline$\Delta$, deg & \multicolumn{1}{c}{ TJ } & \multicolumn{1}{c}{$68 \mathrm{M}$} & \multicolumn{1}{c}{ C2 } & Error \\
\hline 30 & $181.9 \pm 0.4$ & $181.6 \pm 0.6$ & 181.3 & 0.3 \\
35 & $151.4 \pm 0.3$ & $151.6 \pm 0.6$ & 150.8 & 0.8 \\
40 & $125.1 \pm 0.5$ & $124.6 \pm 0.6$ & 123.6 & 1.0 \\
45 & $100.7 \pm 0.4$ & $100.5 \pm 0.6$ & 99.7 & 0.8 \\
50 & $79.9 \pm 0.4$ & $79.6 \pm 0.6$ & 79.0 & 0.6 \\
55 & $62.3 \pm 1.0$ & $61.5 \pm 0.6$ & 61.2 & 0.3 \\
60 & $46.1 \pm 1.0$ & $45.9 \pm 0.6$ & 45.9 & 0.0 \\
65 & $33.0 \pm 1.0$ & $32.8 \pm 0.6$ & 32.9 & -0.1 \\
70 & $22.0 \pm 2.7$ & $22.0 \pm 0.6$ & 22.4 & -0.4 \\
75 & $13.4 \pm 2.1$ & $13.5 \pm 0.6$ & 14.1 & -0.6 \\
80 & & $7.2 \pm 0.6$ & 7.7 & -0.5 \\
85 & & $3.1 \pm 0.6$ & 3.4 & -0.3 \\
90 & & $1.1 \pm 0.6$ & 1.2 & -0.1 \\
\hline
\end{tabular}

TJ denotes Jordan [1973], and 68M denotes Engdahl and Johnson [1974].
TABLE 10. PKP Times (Surface Focus) and Errors

\begin{tabular}{|c|c|c|c|c|c|c|c|}
\hline \multirow[b]{2}{*}{$\Delta, \operatorname{deg}$} & \multirow[b]{2}{*}{ JB } & \multirow[b]{2}{*}{68} & \multirow[b]{2}{*}{ JW } & \multirow[b]{2}{*}{$\mathrm{C} 2$} & \multicolumn{3}{|c|}{ Difference } \\
\hline & & & & & JB & 68 & JW \\
\hline $170 \mathrm{~A}$ & 1286.3 & 1283.7 & & 1284.7 & 1.6 & -1.0 & \\
\hline 160 & 1242.7 & 1239.7 & 1241.8 & 1240.5 & 2.2 & -0.8 & 1.3 \\
\hline 150 & 1200.2 & 1196.9 & 1199.2 & 1197.7 & 2.5 & -0.8 & 1.5 \\
\hline 145B & 1180.4 & 1178.0 & 1179.4 & 1177.7 & 2.7 & +0.3 & 1.7 \\
\hline $145 B$ & 1179.3 & 1174.4 & 1178.9 & 1176.7 & 2.6 & -2.3 & 2.2 \\
\hline 150 & $\cdots$ & 1188.1 & 1192.6 & 1190.1 & $\cdots$ & -2.0 & 2.5 \\
\hline $155 \mathrm{C}$ & $\cdots$ & 1201.0 & 1204.1 & 1201.4 & . & -0.4 & 2.7 \\
\hline $122 \mathrm{D}$ & & & 1136.8 & 1134.7 & & & 2.1 \\
\hline 125 & & & 1142.7 & 1140.5 & & & 2.2 \\
\hline 130 & 1152.0 & 1151.3 & 1152.5 & 1150.2 & 1.8 & 1.1 & 2.3 \\
\hline 140 & 1170.5 & 1170.1 & 1171.2 & 1169.3 & 1.2 & 0.8 & 2.1 \\
\hline 150 & 1187.4 & 1186.8 & 1188.0 & 1185.9 & 1.5 & 0.9 & 2.1 \\
\hline 160 & 1200.8 & 1200.0 & 1201.5 & 1199.3 & 1.5 & 0.7 & 2.2 \\
\hline 170 & 1209.2 & 1208.4 & 1210.4 & 1208.3 & 1.1 & 0.1 & 2.1 \\
\hline $180 \mathrm{~F}$ & 1212.2 & 1211.0 & 1213.6 & 1211.8 & 0.4 & -0.8 & 1.8 \\
\hline $\begin{array}{l}\text { erage } \\
\text { difference }\end{array}$ & & & & & +1.7 & -0.3 & +2.1 \\
\hline$P_{\mathrm{AB}}{ }^{\prime}-P_{\mathrm{DF}}^{\prime}$ & & & & & & & \\
\hline 170 & 77.1 & 75.3 & .. & 76.4 & 0.7 & -1.1 & $\cdots$ \\
\hline 160 & 41.9 & 39.7 & 40.3 & 41.2 & 0.7 & -1.5 & -0.9 \\
\hline 150 & 12.8 & 10.1 & 11.2 & 11.8 & 1.0 & -1.7 & -0.6 \\
\hline$P_{\mathrm{BC}}{ }^{\prime}-P_{\mathrm{DF}^{\prime}}, 150$ & $0 \ldots$ & 1.3 & 4.6 & 4.2 & ... & -2.9 & +0.4 \\
\hline
\end{tabular}

JB denotes Jeffreys and Bullen [1940]; 68, Herrin et al. [1968]; and JW, Whitcomb [1973].

wave travel times are bracketed by body wave solutions. Outside these regions, $\mathrm{C} 2$ is at least $2.2 \mathrm{~s}$ slow in comparison with body wave data. The calculated $d t / d A$ for shear waves is generally consistent with observed values except possibly between $45^{\circ}-50^{\circ}$. This is the region where data of Hart [1975] indicates revisions of earlier solutions.

$S c S$ times are even less studied than $S$ times. Model C2 is 6.0 s slower than JB or Gogna times. This is consistent with C2 $S$ times, which are 4.5-5.9 slower than JB and Gogna times. The $S$ and $S c S$ data are therefore reasonably consistent with the view that average shear wave travel times in the mantle are about $4 \mathrm{~s}$ slower than standard body wave solutions. This can be compared with the earlier conclusions that the average earth is $0.6 \mathrm{~s}$ slower for $\boldsymbol{P}$ waves than obtained for that part of the earth available for $P$ wave inspection.

The scatter in measured $S c S-S$ times is 5-10 s [Hales and Roberts, 1970; Jordan, 1972; Jordan and Lynn, 1974]. This has been attributed to lateral variations in mantle $S$ times [Jordan and Lynn, 1974] deep in the mantle. The average residual $\left(S_{C} S\right.$ -

TABLE 11. PKiKP-PCP Times

\begin{tabular}{cccc}
\hline$\Delta$, deg & EFM & C2 & Error \\
\hline 10.90 & 477.5 & 478.3 & -0.8 \\
11.73 & 477.2 & 477.6 & -0.4 \\
21.34 & 464.9 & 466.3 & -1.4 \\
26.64 & 457.4 & 457.7 & -0.3 \\
27.71 & 454.8 & 455.7 & -0.9 \\
29.69 & 451.2 & 452.1 & -0.9 \\
30.50 & 450.4 & 450.5 & -0.1 \\
30.60 & 449.5 & 450.3 & -0.8 \\
31.08 & 448.2 & 449.3 & -1.1 \\
35.94 & 438.4 & 439.1 & -0.7 \\
36.04 & 438.8 & 438.9 & -0.1 \\
38.17 & 433.5 & 433.3 & +0.2 \\
47.18 & 411.9 & 412.1 & -0.2 \\
Mean error & & & -0.6 \\
\hline
\end{tabular}

Values are in seconds. EFM denotes Engdahl et al. [1974]. 
TABLE 12. Shear Wave Travel Times (Surface Focus) and $d t / d \Delta$

\begin{tabular}{cccccccc}
\hline & & & & & \multicolumn{3}{c}{$d t / d \Delta, \mathrm{s} / \mathrm{deg}$} \\
\cline { 5 - 8 } $\begin{array}{c}\Delta, \\
\text { deg }\end{array}$ & \multicolumn{1}{c}{ JB } & FEF & HR & C2 & HR & FEF & C2 \\
\hline 30 & 670.2 & 680.0 & 669.5 & 676.7 & 15.4 & 16.0 & 15.5 \\
35 & 748.2 & 757.2 & 749.0 & 753.1 & 15.3 & 15.3 & 15.2 \\
40 & 824.5 & 831.5 & 825.7 & 821.1 & 15.2 & 14.6 & 14.9 \\
45 & 897.9 & 902.3 & 899.5 & 902.4 & 14.5 & 14.1 & 14.8 \\
50 & 968.6 & 972.5 & 970.5 & 975.0 & 13.9 & 13.8 & 14.5 \\
55 & 1036.8 & 1041.1 & 1038.7 & 1043.2 & 13.4 & 13.3 & 13.4 \\
60 & 1102.6 & 1106.5 & 1104.1 & 1109.2 & 12.8 & 12.9 & 13.0 \\
65 & 1165.5 & 1169.5 & 1166.7 & 1172.2 & 12.2 & 12.3 & 12.1 \\
70 & 1225.6 & 1229.9 & 1226.4 & 1231.4 & 11.7 & 11.9 & 11.6 \\
75 & 1282.6 & 1288.1 & 1283.2 & 1288.1 & 11.1 & 11.1 & 11.1 \\
80 & 1336.5 & 1341.9 & 1337.3 & 1342.1 & 10.5 & 10.3 & 10.6 \\
85 & 1387.3 & 1391.2 & 1388.5 & 1393.3 & 10.0 & 9.7 & 9.9 \\
90 & 1435.5 & 1438.9 & 1436.9 & 1441.1 & 9.4 & 9.2 & 9.2 \\
95 & 1478.2 & 1484.0 & 1482.4 & 1486.0 & 8.8 & 8.8 & 8.8 \\
\hline
\end{tabular}

JB denotes Jeffreys and Bullen [1940]; FEF, F. E. Followill and O. Nuttli (personal communication, 1971); and HR, Hales and Roberts [1970].

$S)_{\mathrm{C} 2}-(S c S-S)_{\mathrm{JB}}$ over the distance range $30^{\circ}-80^{\circ}$ is $+0.7 \mathrm{~s}$ for deep focus events. The Jordan [1972] data set gives $+1.7 \pm 1.3$ s $\left(95 \%\right.$ confidence interval). Between $40^{\circ}$ and $70^{\circ}, \mathrm{C} 2$ has a JB residual of $+0.4 \mathrm{~s}$ compared with the Jordan [1972] value +0.5 s. The $S c S-S$ data are summarized in Table 13. We conclude that $\mathrm{C} 2$ is an adequate fit to the $S c S-S$ data.

\section{Composition of the Mantle}

Burdick and Anderson [1975] showed that the major features of the upper mantle and transition region could be accounted for by an olivine-rich mantle undergoing phase changes to $\beta$ spinel near $400 \mathrm{~km}$ and $\gamma$ spinel near $500 \mathrm{~km}$. Some pyroxene was required in order to satisfy the densities and to make the fayalite content of the olivine more in line with petrologic estimates. The mantle between the base of the $\mathrm{LVZ}$ and 600 $\mathrm{km}$ appeared to be chemically homogeneous. The $650-\mathrm{km}$ discontinuity appears to be the result of the transformation of pyroxene-garnet to oxides or perovskite (D. L. Anderson, in preparation, 1976). The lower mantle has properties consistent with the properties of the mixed oxides $(\mathrm{Fe}, \mathrm{Mg}) \mathrm{O}+\mathrm{SiO}_{2}$ (stishovite). Anderson and Sammis [1970] concluded from earlier earth models that the $\mathrm{LVZ}$ was due to partial melting and that small amounts of water were required in order to depress the melting point. All of these conclusions are consistent with the new earth model. The structure below $670 \mathrm{~km}$ indicates further phase changes, possibly involving

TABLE 13. $S c S-S$ Travel Times

\begin{tabular}{cccc}
\hline$\Delta$, deg & JA* & C2 & Difference \\
\hline 30 & $311.3 \pm 1.8$ & 306.8 & -4.5 \\
35 & $259.4 \pm 1.5$ & 258.3 & -1.1 \\
40 & $215.7 \pm 1.6$ & 213.3 & -2.4 \\
45 & $174.3 \pm 1.1$ & 172.2 & -2.1 \\
50 & $138.6 \pm 1.4$ & 137.9 & -0.8 \\
55 & $108.5 \pm 1.3$ & 107.2 & -1.3 \\
60 & $82.0 \pm 1.1$ & 80.7 & -1.3 \\
65 & $59.7 \pm 0.9$ & 59.2 & -0.5 \\
70 & $40.6 \pm 1.0$ & 41.2 & -0.6 \\
75 & $25.5 \pm 1.3$ & 26.6 & 1.1 \\
80 & $14.0 \pm 0.8$ & 15.1 & 1.1 \\
\hline
\end{tabular}

JA denotes Jordan and Anderson [1974].

"Uncertainty is $95 \%$ confidence interval. ilmenite, perovskite, and mixed oxide assemblages. The lower mantle between depths of about 1500 and $2600 \mathrm{~km}$ is relatively homogeneous. Velocity gradients, and possibly the density gradient, in the lowermost $300 \mathrm{~km}$ of the mantle are less than in the rest of the lower mantle. This could be due to temperature or compositional gradients in this region. A high temperature gradient could result from heating by the core. The core efficiently brings heat to the base of the mantle both by conduction and by convection, but heat is not easily transported across the boundary because of the more insulating nature of silicates. A high temperature gradient at the base of the mantle is therefore to be expected. The $U$ and Th content at the base of the mantle may also be high [Anderson, 1972, 1975; Anderson and Hanks, 1972]. There is also the possibility that the bulk chemistry of this region is more refractory than is that of the normal mantle [Anderson, 1972].

The uppermost mantle, the lid of the low-velocity zone, does not fall on any reasonable extrapolation of the 250 - to $300-\mathrm{km}$ region of the mantle. The velocities in the lid are greater than in olivine or pyroxene and must contain substantial portions of spinel or garnet or some other dense phase. The velocities and density are appropriate for eclogite. The closest match is with an eclogite from Nordfjord, Norway, which has $24 \%$ orthopyroxene, $23 \%$ clinopyroxene, and $51 \%$ garnet [Manghnani et al., 1974]. Press [1969] has also suggested that eclogite may be an important component of the upper mantle. These conclusions are based partly on the evidence from body wave studies for high velocities in the lithosphere, since the normal mode data set has limited resolving power in this region of the mantle.

\section{SUMMARY}

An earth model based on high-resolution body wave studies has been inverted with the use of a representative set of 400 normal mode periods including many higher modes. The resulting model, designated $\mathrm{C} 2$, satisfies the free oscillation data with an average error of about $0.08 \%$. It is also in agreement with a large body of travel time, apparent velocity, and differential travel time data. Although there is a large spread in body wave solutions, there is a suggestion that the average earth, mainly oceans, is slightly slower than that part of the earth available for body wave inspection, mainly tectonic to continental paths.

Model C2 has pronounced low-velocity zones for both $P$ and $S$, a relatively high-density and high-velocity upper mantle lid, and transition regions near $375-425,500-550$, and $650-675$ $\mathrm{km}$. There is also moderate structure between 700 and $1200 \mathrm{~km}$ and slight inhomogeneity on both sides of the mantle-core and outer core-inner core boundaries. The $V_{p}$ and density jump at the outer core-inner core boundary is small. The radius of the inner core is probably slightly larger than the $1215 \mathrm{~km}$ given by the model.

The uppermost mantle is consistent with eclogite overlying a thick partially molten zone. The rest of the upper mantle is consistent with olivine and pyroxene and their successive transformation to higher-pressure assemblages.

Acknowledgments. This research was supported by the Advanced Research Projects Agency of the Department of Defense and was monitored by the Air Force Office of Scientific Research under contract F44620-72-C-0078. Tom Jordan participated extensively in the early part of this study, and we are indebted to him for much advice. We would like to thank F. Gilbert, A. Dziewonski, D. Helmberger, and B. Bolt for preprints of their papers. Conversations with Martin Smith and Freeman Gilbert were quite helpful. One of us (R.S.H.) thanks the National Science Foundation for graduate fellowship 
support. Contribution 2637 of the Division of Geological and Planetary Sciences, California Institute of Technology, Pasadena, California 91125 .

\section{REFERENCES}

Anderson, D. L., Phase changes in the upper mantle, Science, 157. $1165,1967 a$.

Anderson, D. L., Latest information from seismic observations, The Earth's Mantle, edited by T. F. Gaskell, p. 355, Academic, New York, $1967 b$.

Anderson, D. L., Implications of the inhomogeneous planetary accretion hypothesis, Comments Earth Sci. Geophys. 2, 93, 1972.

Anderson, D. L., Chemical plumes in the mantle, Geol. Soc. Amer. Bull., 86, 1593, 1975.

Anderson, D. L., and T. C. Hanks, Formation of the Earth's core, Nature, 237, 387, 1972.

Anderson, D. L., and C. Sammis, Partial melting in the upper mantle, Phys. Earth Planet. Interiors, 3, 41, 1970.

Anderson, D. L., and M. N. Toksöz, Surface waves on a spherical earth, 1, Upper mantle structure from Love waves, J. Geophys. Res., $68,3483,1963$.

Backus, G., and F. Gilbert, The resulting power of grass earth data, Geophys. J. Roy. Astron. Soc., 16, 169-205, 1968.

Bolt, B. A., and R. G. Currie, Maximum entropy estimates of Earth torsional eigenperiods from 1960 Trieste data, Geophys. J. Roy. Astron. Soc., 40, 107, 1975.

Bolt, B. A., M. Niazi, and M. Sommerville, Diffracted ScS and the shear velocity at the core boundary, Geophys. J. Roy. Astron. Soc., $19,299,1970$.

Brune, J. N., and F. Gilbert, Torsional overtone dispersion from correlations of $S$ waves to $S S$ waves, Bull. Seismol. Soc. Amer., 64, $313,1974$.

Burdick, L. J., and D. L. Anderson, Interpretation of velocity profiles of the mantle, J. Geophys. Res., 80, 1070, 1975.

Carder, D. S., D. W. Gordon, and J. N. Jordan, Analysis of surfacefoci travel times, Bull. Seismol. Soc. Amer., 56, 815, 1966.

Cleary, J. R., and A. L. Hales, An analysis of the travel times of $P$ waves to North American stations in the distance range of $32^{\circ}$ to $100^{\circ}$, Bull. Seismol. Soc. Amer., 56, 467, 1966.

Corbishley, D. J., Multiple array measurements of the $\boldsymbol{P}$-wave traveltime derivative, Geophys. J. Roy. Astron. Soc., 19, 1, 1970.

Derr, J. S., Free oscillation observations through 1968, Bull. Seismol. Soc. Amer., 59, 2079, 1969.

Doyle, H. A., and A. L. Hales, An analysis of the travel times of $S$ waves to North American stations in the distance range $28^{\circ}$ to $82^{\circ}$, Bull. Seismol. Soc. Amer., 57, 761, 1967.

Dziewonski, A., On regional differences in dispersion of mantle Rayleigh waves, Geophys. J. Roy. Astron. Soc., 22, 289, 1971.

Dziewonski, A., and F. Gilbert, Observations of normal modes from 84 recordings of the Alaskan earthquake of 1964, March 28, Geophys. J. Roy. Astron. Soc., 27, 393, 1973.

Dziewonski, A., J. Mills, and S. Bloch, Residual dispersion measurement-A new method of surface wave analysis, Bull. Seismol. Soc. Amer., 62, 129, 1972.

Dziewonski, A., A. L. Hales, and E. R. Lapwood, Parametrically simple earth models consistent with geophysical data, Phys. Earth Planet. Interiors, 10, 12, 1975.

Engdahl, E. R., and E. A. Flınn, Seismic waves reflected from discontinuities within the upper mantle, Science, 163, 177, 1969.

Engdahl, E. R., and L. E. Johnson, Differential $P c P$ travel-times and the radius of the core, Geophys. J. Roy. Astron. Soc., 39, 435, 1974.

Engdahl, E. R., E. A. Flinn, and R. P. Masse, Differential PKiKP travel-times and the radius of the inner core, Geophys. J. Roy. Astron. Soc., 39, 457, 1974

Gilbert, F., and A. Dziewonski, An application of normal mode theory to the retrieval of structural parameters and source mechanisms from seismic spectra, Phil. Trans. Roy. Soc. London. Ser. A, 278, 187, 1975 .

Gilbert F., A. Dziewonski, and J. Brune, An informative solution to a seismological inverse problem, Proc. Nat. Acad. Sci. U.S. A., 70, 1410-1413, 1973.

Gogna, M. L., Travel times of $S, P c P$, and $S c S$ from Pacific earthquakes, Geophys. J. Roy. Astron. Soc., 33, 103, 1973.
Hales, A. L., Eigenperiods of earth models and the determination of travel-time baselines, J. Geophys. Res., 79, 422, 1974.

Hales, A. L., and J. L. Roberts, Shear velocities in the lower mantle and the radius of the core, Bull. Seismol. Soc. Amer., 60, 1427, 1970.

Hales, A. L., J. R. Cleary, and J. L. Roberts, Velocity distributions in the lower mantle, Bull. Seismol. Soc. Amer., 58, 1975, 1968.

Hart, R. S., Shear velocity in the lower mantle from explosion data, $J$. Geophys. Res., 80, 4889, 1975.

Hart, R. S., and F. Press, $S_{n}$ velocities and the composition of the lithosphere in the regionalized Atlantic, J. Geophys. Res., 78, $407-411,1973$.

Helmberger, D. V., and G. R. Engen, Upper mantle shear structure, $J$. Geophys. Res., 79, 4017, 1974.

Helmberger, D. V., and R. A. Wiggins, Upper mantle structure of middlewestern United States, J. Geophys. Res., 76, 3229, 1971.

Herrin, E. (chairman), 1968 seismological tables for $\boldsymbol{P}$ phases, Bull. Seismol. Soc. Amer., 58, 1193, 1968.

Ibrahim, A. K., and O. Nuttli, Travel-time curves and upper mantle structure from long period $S$ waves, Bull. Seismol. Soc. Amer., 57, $1063,1967$.

Jeffreys, H., and Bullen, K. E., Seismological Tables, 55 pp., British Association for the Advancement of Science, London, 1940.

Johnson, L. R., Array measurements of $\boldsymbol{P}$ velocities in the upper mantle, J. Geophys. Res., 72, 6309-6325, 1967.

Jordan, T. H., Estimation of the radial variation of seismic velocities and density in the earth, Ph.D. thesis, Calif. Inst. of Technol, Pasadena, 1972.

Jordan, T. H., and D. L. Anderson, Earth structure from free oscillations and travel times, Geophys. J. Roy. Astron. Soc., 36, 411, 1974.

Jordan, T. H., and W. S. Lynn, A velocity anomaly in the lower mantle, J. Geophys. Res., 79, 2679, 1974.

Julian, B. R., and D. L. Anderson, Travel times, apparent velocities and amplitudes of body waves, Bull. Seismol. Soc. Amer., 58 , $339-366,1968$.

Kanamori, H., Velocity and $Q$ of mantle waves, Phys. Earth Planet. Interiors, 2, 259, 1970.

Kogan, S. D., Travel times of longitudinal and transverse waves calculated from data on nuclear explosions made in the region of the Marshall Islands, Izv. Acad. Sci. USSR Phys. Solid Earth, Engl. Transl., no. 3, 242, 1960.

Kosminskaya, I. P., N. N. Puzyrev, and A. S. Aleks-eyev, Explosion seismology: Its past, present and future, Tectonophysics, 13, 309$324,1972$.

Manghnani, M. H., R. Ramananantóandro, and S. P. Clark, Compressional and shear wave velocities in granulite facies rocks and eclogites to 10 kbar, J. Geophys. Res., 79, 5427, 1974.

Mendiguren, J. A., Identification of the free oscillation spectra peaks for 1970 July 31 , Columbian deep shock using the excitation criterion, Geophys. J. Roy. Astron. Soc., 33, 281, 1973.

Müller, G., Amplitude studies of core phases, J. Geophys. Res., 78, $3469,1973$.

Niazi, M., and D. L. Anderson, Upper mantle structure of western North America from apparent velocities of $\boldsymbol{P}$ waves, $J$. Geophys. Res., 70, 4633, 1965.

Niazi, M., $S H$ travel times and lateral heterogeneities in the lower mantle, Bull. Seismol. Soc. Amer., 63, 2035, 1973.

Press, F., The suboceanic mantle, Science, 165, 174-176, 1969.

Simpson, D. W., $P$ wave velocity structure of the upper mantle in the Australian region, Ph.D. thesis, Aust. Nat. Univ., Canberra, 1973.

Sutton, G. H., and D. A. Walker, Oceanic mantle phases recorded on seismographs in the northwestern Pacific at distances between $7^{\circ}$ and $40^{\circ}$, Bull. Seismol. Soc. Amer., 62, 631, 1972.

Whitcomb, J. H., A study of the velocity structure of the Earth by the use of core phases, I, Ph.D. thesis, Calif. Inst. of Technol., Pasadena, 1973 .

Whitcomb, J. H., and D. L. Anderson, Reflection of $P^{\prime} P^{\prime}(P K P P K P)$ seismic waves from discontinuities in the mantle, J. Geophys. Res., $75,5713,1970$

(Received June 9, 1975;

revised October 14, 1975; accepted November 7,1975 .) 